\title{
Dark Energy Survey Year 3 Results: Deep Field optical + near-infrared images and catalogue
}

W. G. Hartley, ${ }^{1 \star}$ A. Choi, ${ }^{2 \star}$ A. Amon, ${ }^{3 \star}$ R. A. Gruendl, ${ }^{4,5}$ E. Sheldon, ${ }^{6}$ I. Harrison, ${ }^{7,8}$ G. M. Bernstein, ${ }^{9}$ I. Sevilla-Noarbe, ${ }^{10}$ B. Yanny, ${ }^{11}$ K. Eckert, ${ }^{9}$ H. T. Diehl, ${ }^{11}$ A. Alarcon, ${ }^{12}$ M. Banerji, ${ }^{13,14}$ K. Bechtol, ${ }^{15}$ R. Buchs, ${ }^{16}$ S. Cantu, ${ }^{17}$ C. Conselice,${ }^{8,18}$ J. Cordero, ${ }^{8}$ C. Davis,${ }^{3}$ T. M. Davis, ${ }^{19}$ S. Dodelson, ${ }^{20}$

A. Drlica-Wagner, ${ }^{21,11,22}$ S. Everett, ${ }^{23}$ A. Ferté, ${ }^{24}$ D. Gruen, ${ }^{3,16,25}$ K. Honscheid, ${ }^{2,26}$ M. Jarvis, ${ }^{9}$ M. D. Johnson, ${ }^{5}$ N. Kokron, ${ }^{3,25}$ N. MacCrann, ${ }^{27}$ J. Myles, ${ }^{3,16,25}$ A. B. Pace, ${ }^{20}$ A. Palmese, ${ }^{11,22}$

F. Paz-Chinchón, ${ }^{5,13}$ M. E. S. Pereira, ${ }^{28}$ A. A. Plazas, ${ }^{29}$ J. Prat, ${ }^{21}$ M. Rodriguez-Monroy, ${ }^{10}$ E. S. Rykoff,, 316 S. Samuroff, ${ }^{20}$ C. Sánchez, ${ }^{9}$ L. F. Secco,${ }^{9}$ F. Tarsitano, ${ }^{30}$ A. Tong,${ }^{31}$ M. A. Troxel,${ }^{31}$ Z. Vasquez, ${ }^{20}$

K. Wang, ${ }^{31}$ C. Zhou, ${ }^{31}$ T. M. C. Abbott, ${ }^{32}$ M. Aguena, ${ }^{33,34}$ S. Allam, ${ }^{11}$ J. Annis, ${ }^{11}$ D. Bacon, ${ }^{35}$

E. Bertin, ${ }^{36,37}$ S. Bhargava, ${ }^{38}$ D. Brooks, ${ }^{39}$ D. L. Burke, ${ }^{3,16}$ A. Carnero Rosell, ${ }^{40,41}$ M. Carrasco Kind, ${ }^{4,5}$ J. Carretero, ${ }^{42}$ F. J. Castander, ${ }^{43,44}$ M. Costanzi, ${ }^{45,46}$ M. Crocce,${ }^{43,44}$ L. N. da Costa, ${ }^{34,47}$ J. De Vicente, ${ }^{10}$ J. DeRose, ${ }^{23,48}$ S. Desai, ${ }^{49}$ J. P. Dietrich,${ }^{50}$ T. F. Eifler, ${ }^{24,51}$ J. Elvin-Poole, ${ }^{2,26}$ I. Ferrero, ${ }^{52}$ B. Flaugher, ${ }^{11}$ P. Fosalba, ${ }^{43,44}$ J. García-Bellido, ${ }^{53}$ E. Gaztanaga, ${ }^{43,44}$ D. W. Gerdes,${ }^{28,54}$ J. Gschwend, ${ }^{34,47}$ G. Gutierrez, ${ }^{11}$ S. R. Hinton, ${ }^{19}$ D. L. Hollowood, ${ }^{23}$ D. Huterer, ${ }^{28}$ D. J. James, ${ }^{55}$ S. Kent, ${ }^{11,22}$ E. Krause, ${ }^{51}$ K. Kuehn, ${ }^{56,57}$ N. Kuropatkin, ${ }^{11}$ O. Lahav, ${ }^{39}$ H. Lin, ${ }^{11}$ M. A. G. Maia, ${ }^{34,47}$ M. March, ${ }^{9}$ J. L. Marshall, ${ }^{17}$ P. Martini, ${ }^{2,58,59}$ P. Melchior, ${ }^{29}$ F. Menanteau, ${ }^{4,5}$ R. Miquel, ${ }^{42,60}$ J. J. Mohr, ${ }^{50,61}$ R. Morgan, ${ }^{15}$ E. Neilsen, ${ }^{11}$ R. L. C. Ogando, ${ }^{34,47}$ S. Pandey, ${ }^{9}$ A. K. Romer, ${ }^{38}$ A. Roodman, ${ }^{3,16}$ M. Sako, ${ }^{9}$ E. Sanchez, ${ }^{10}$ V. Scarpine, ${ }^{11}$ S. Serrano, ${ }^{43,44}$ M. Smith, ${ }^{62}$ M. Soares-Santos, ${ }^{28}$ E. Suchyta, ${ }^{63}$ M. E. C. Swanson,,${ }^{5}$ G. Tarle,${ }^{28}$ D. Thomas, ${ }^{35}$ C. To, ${ }^{25,3,16}$ T. N. Varga, ${ }^{61,64}$ A. R. Walker, ${ }^{32}$ W. Wester, ${ }^{11}$ R.D. Wilkinson, ${ }^{38}$ and J. Zuntz ${ }^{65}$ (DES Collaboration)

Affiliations are listed at the end of the paper

Accepted 2021 October 12. Received 2021 October 11; in original form 2021 January 5

\begin{abstract}
We describe the Dark Energy Survey (DES) Deep Fields, a set of images and associated multiwavelength catalogue (ugrizJHKs) built from Dark Energy Camera (DECam) and Visible and Infrared Survey Telescope for Astronomy (VISTA) data. The DES Deep Fields comprise 11 fields (10 DES supernova fields plus COSMOS), with a total area of $~ 30 \mathrm{sq}$. deg. in ugriz bands and reaching a maximum $i$-band depth of 26.75 (AB, 10 $\sigma, 2$ arcsec). We present a catalogue for the DES 3-yr cosmology analysis of those four fields with full 8-band coverage, totalling $5.88 \mathrm{sq}$. deg. after masking. Numbering 2.8 million objects (1.6 million post-masking), our catalogue is drawn from images coadded to consistent depths of $r=25.7, i=25$, and $z=24.3$ mag. We use a new model-fitting code, built upon established methods, to deblend sources and ensure consistent colours across the $u$-band to $K s$-band wavelength range. We further detail the tight control we maintain over the point-spread function modelling required for the model fitting, astrometry and consistency of photometry between the four fields. The catalogue allows us to perform a careful star-galaxy separation and produces excellent photometric redshift performance (NMAD $=0.023$ at $i<23$ ). The Deep-Fields catalogue will be made available as part of the cosmology data products release, following the completion of the DES 3-yr weak lensing and galaxy clustering cosmology work.
\end{abstract}

Key words: catalogues - surveys - galaxies: distances and redshifts - galaxies: evolution-galaxies: photometry-cosmology: observations. 


\section{INTRODUCTION}

Deep survey fields have long been a mainstay of optical extragalactic astronomy (Kron 1980; Williams et al. 1996; Wittman et al. 2002; Kashikawa et al. 2004; Scoville et al. 2007; Furusawa et al. 2008). Given a limited amount of observing time, there is a necessary tradeoff between survey depth and area. Studies of the most distant galaxies and faintest objects require images of extremely high sensitivity, which are expensive to build up and, as a result, the deepest fields typically cover just a few square degrees or smaller. Nevertheless, a square degree of area is sufficient to reduce sample variance uncertainties to acceptable levels in most studies of galaxy evolution at moderate-and-high redshifts (Somerville et al. 2004). ${ }^{1}$ Among the great many uses of these deep fields are statistical studies such as galaxy luminosity and stellar mass functions (e.g. Cohen 2002; Mortlock et al. 2015), galaxy biasing (e.g. Coil et al. 2004; Hartley et al. 2013), morphological properties of intermediate and high-redshift galaxies (e.g. Lotz et al. 2006; Elmegreen et al. 2007) and searches for peculiar or extreme sub-populations (e.g. Daddi et al. 2004; Bouwens et al. 2007).

Over the decades of work using deep extragalactic fields, our understanding of distant galaxy populations has been accelerated by the addition of longer wavelength data (Lonsdale et al. 2003; Foucaud et al. 2007; Lawrence et al. 2007; Koekemoer et al. 2011; McCracken et al. 2012; Jarvis et al. 2013) and spectroscopy (Le Fèvre et al. 2005; Lilly et al. 2007; Coil et al. 2011; Bradshaw et al. 2013; McLure et al. 2013, 2018; Le Fèvre et al. 2015), which provide complementary information on the properties of the surveyed galaxies. Near-infrared data in particular are crucial for detecting galaxies at $z>1$, where even massive galaxies can drop out of optical images if they lack significant star formation. In combination, surveys at differing wavelength ranges are of far greater value than the sum of their parts, and as such the fields where these data already exist become the natural targets of further observations. We now have around half a dozen of these deep survey fields scattered across the extragalactic sky, containing panchromatic imaging data and abundant spectroscopy. Key amongst them are the Cosmological Evolution Survey (COSMOS; Scoville et al. 2007)/UltraVISTA (McCracken et al. 2012), and Subaru/XMM-Newton Deep Survey (SXDS; Furusawa et al. 2008)/UKIDSS Ultra-Deep Survey (UDS; Almaini et al., in preparation) equatorial fields, and the Great Observatories Origins Deep Survey South (GOODS-S; Giavalisco et al. 2004) field at declination, $\delta \sim-28$.

Among the images that have been built up over the last ten years in the key extragalactic fields are those collected using dedicated survey instruments or facilities, e.g. Wide-Field Camera (WFCam) on the UK InfraRed Telescope (UKIRT), the Visible and Infrared Survey Telescope for Astronomy (VISTA; Emerson et al. 2004), and Hyper SuprimeCam (HSC; Aihara et al. 2018). In such cases, the varied science goals envisioned for these instruments leads to a 'weddingcake' strategy, of multiple sub-surveys making different area-depth trade-offs (Lawrence et al. 2007; Aihara et al. 2018). A further benefit of this type of survey strategy is that the deeper and richer survey fields can be used to better understand the shallower but wider-area survey, for instance by providing redshift information for colourselected samples (e.g. Kim et al. 2011), or a true source list when estimating detection completeness (Sevilla-Noarbe et al. 2021). In

\footnotetext{
${ }^{1}$ Where sample variance is a concern, it is usually more efficiently overcome by similar-sized independent, widely separated fields, rather than through expanding area in one or two existing regions of the sky.
}

this paper, we describe the homogeneous set of images that we have constructed across 11 deep fields, and the catalogue derived from four of them in order to understand and overcome sources of systematic uncertainty in the Dark Energy Survey (DES; Flaugher 2005; DES Collaboration 2016; Lahav et al. 2020) three-year (Y3) cosmology analysis. Although developed with this specific use in mind, we also detail our careful star-galaxy separation and photometric redshifts that make the resulting catalogue a cutting edge data product for a broad range of extragalactic astronomy.

The DES has as its primary goal the characterization of dark energy through measuring the expansion history of the Universe and growth of structure at late times. The principal probes used by the survey team are weak gravitational lensing, galaxy clustering (including the baryon acoustic oscillation scale length), abundance of galaxy clusters and light curves of distant type-1a supernovae (SN). The survey includes a main wide-field survey of the southern Galactic cap (Abbott et al. 2018) and a cadenced sub-survey optimised for SN detection (D'Andrea et al. 2018). Observations were made using the Dark Energy Camera (DECam; Flaugher et al. 2015), mounted on the Victor M. Blanco 4-m telescope at the Cerro Tololo InterAmerican Observatory near La Serena, Chile. DES observations began in 2013 August (Y1) and continued for five subsequent Southern hemisphere spring and summer seasons until completion in 2019 January. The wide survey (WS) spans $5100 \mathrm{deg}^{2}$ and comprises 10 sets of exposures per photometric band $(g, r, i, z$, and $Y)$ that each tile the entire footprint. Tilings are offset by up to half of the 1-deg field diameter to fill focal-plane gaps and strongly tie the exposures' photometric calibration, such that a typical source is imaged 8 times per filter.

Concurrently, the SN sub-survey of ten fields was carried out with roughly weekly cadence, and sometimes chosen when observing conditions yielded a point-spread function (PSF) unacceptable for the $\mathrm{WS}^{2}$ (Neilsen et al. 2019). As the goal of this sub-survey was to detect and characterize SN light curves, observations were minimally dithered. During the five years that this sub-survey was active, roughly 30 per cent of the total observing time was taken in these 10 supernova pointings. The locations of the SN fields lie within the main survey footprint and were chosen to coincide with a number of pre-existing deep extragalactic survey fields, particularly the VISTA Deep Extragalactic Observations survey (VIDEO; Jarvis et al. 2013) and spectroscopic surveys. Combined with DECam observations of the COSMOS/UltraVISTA field, the SN fields have already provided high-quality redshift information for photometric redshift (photo-z) calibration in DES (Sánchez et al. 2014; Bonnett et al. 2016, Hoyle et al. 2018).

In the DES Y3 weak lensing and clustering cosmology analysis, the DES Deep Fields (SN fields plus COSMOS) are used to understand sources of systematic uncertainties in the main survey area and how they propagate through the cosmology analysis. Sources measured in the deep stacked coadd images act as an effective truth table of the galaxy population that is present in the main survey, and thus provide a highly accurate input for the 'Balrog' (Suchyta et al. 2016; Everett et al. 2020) and weak-lensing image simulation (MacCrann et al. 2020) pipelines and will, in future, be used in the 'Bayesian Fourier Domain' (BFD; Bernstein \& Armstrong 2014a; Bernstein et al. 2016) shear measurement approach. In the Balrog process, copies of Deep-Fields galaxies are injected into the main survey images and their response to the extraction pipeline measured.

${ }^{2}$ If the exposures had FWHM worse than 1.6 arcsec, the sequence was declared bad and was redone. 
Similarly, the BFD method of measuring weak gravitational lensing (WL) magnification and shear requires a prior on the distribution of galaxy moments in the measurement bands from higher $\mathrm{S} / \mathrm{N}$ images $(\mathrm{S} / \mathrm{N} \geq \sqrt{10} \times$ the summed WS images, see Bernstein et al. 2016).

Furthermore, the inference of galaxy redshift distributions in the DES Y3 analysis relies heavily on the multilayered 'SOMPZ' methodology (Buchs et al. 2019; Sánchez \& Bernstein 2019; Myles et al. 2020). The SOMPZ method is a photometric redshift approach based upon the self-organizing map algorithm (SOM; Kohonen 1982; Masters et al. 2015), and takes as input a set of galaxy photometry. The more photometric bands used as input, the narrower the intrinsic dispersion of galaxy types and redshifts within a single cell of the derived map, and thus the more accurate it is expected to be in terms of mean redshift. To further facilitate the use of the Deep Fields for this purpose, DES and community DECam exposures in complementary bands ( $u$ and $Y$ ) in the SN fields and COSMOS field were also obtained. The final cosmology catalogue contains photometry in eight bands, ugrizJHKs, for 1.6 million sources across four fields with a total area of 5.88 square degrees (after masks are applied).

Similar area and, in some cases, deeper optical data already exist in our Deep Fields. The great value in this work is the ability to combine the WS and Deep Fields data on a consistent footing. Having deep and wide data taken using the same filter bands is essential for the aforementioned Balrog, SOMPZ, and BFD to reach subpercent accuracy. With these goals in mind, we take great care in PSF modelling, photometric calibration with respect to the WS and consistency of tools used, to ensure the highest level of homogeneity between the two data sets that we can achieve.

Though designed with WL cosmology as the primary use, the Deep Fields are anticipated to add to the long tradition of deep extragalactic science performed in these regions of the sky (e.g. Coppin et al. 2006; Williams et al. 2009; Kovač et al. 2010; Ilbert et al. 2013; Lee et al. 2013; Hartley et al. 2015; Galametz et al. 2018; Maltby et al. 2019; Bowler et al. 2020; Momose et al. 2020), and to enhance non-dark energy science in the WS. The ugrizY DECam observations represent an update to the optical data that have previously been available to users of the VIDEO data, while a highly consistent data-set covering both VIDEO and UltraVISTA/COSMOS will open up possibilities to more easily estimate and overcome the sample variance present in studies that have previously relied only on one field. Though often not a major limitation, the sample variance caused by large-scale overdensities in degree-scale fields impacts our understanding of the even basic quantities, such as the stellar mass function at intermediate redshift (Davidzon et al. 2017).

The remainder of the paper is structured as follows: in Section 2, we describe the input images, their processing, creation of coadded images, masking and basic tests of image quality. Catalogue extraction from the detection images is presented in Section 3 and is followed by details of the PSF modelling in Section 3.2. Section 3.3 provides details of the multiband photometry measurements, based on model fits across the $r, i$, and $z$ bands. We then perform a fine-tuning of the photometric zero-points in the four Deep Fields in Section 3.5 and go on to describe our star-galaxy separation and photometric redshifts of the extracted sources in Sections 4 and 5. We conclude in Section 6. In the Appendices we provide additional information about the images and software configurations, an alternative astrometric solution approach and the final catalogue columns. We expect to make this final catalogue available to the community alongside other data products released from the DES Y3 cosmology analysis.

\section{INPUT DATA AND IMAGE CONSTRUCTION}

The scientific utility of deep survey fields is enhanced by the combination of bands covering different wavelengths, both for the DES cosmology analysis and ancillary science. In this section, we describe the construction of the optical DES Deep-Fields images (Section 2.1) and coincident near-infrared images (Section 2.2) that will later be combined in our cosmology catalogue.

\subsection{Optical (DECam) observations}

Construction of the optical-band deep-field images begins with a query to the DECam exposure data base for all images of duration $>30$ s that are within $\approx 1$ arcmin of the nominal field centers of the 10 DES supernova fields (see Fig. 1) in the ugrizY bands. For the griz bands, a very large number of exposures are available from the DES SN search programme, which observes these fields with roughly weekly cadence through their entire periods of visibility. The DES SN program limits dithers to $\$ 20$ arcsec from the nominal pointing, to avoid inhomogeneity of depth or PSF characteristics in the stacked images. Homogeneity is also desirable for the Deep Fields, hence the restriction to well-aligned pointings. DES also conducted single-night observational campaigns in the $u$ band (for all SN fields) and $Y$ band (for a subset of the fields). The DES SN data have been supplemented with all publicly available community observations satisfying the pointing constraints. Community exposures substantially improve the total depth of the $u$-band observations of the SN-E2, SN-C3, and SN-X3 supernova fields. We construct ugrizY images of the COSMOS field from DES and community DECam observations, again constrained to be near the nominal pointing so that stacks of each DECam CCD's exposures yield a homogeneous image (for full details, see Neilsen et al. 2019). A summary for the fields targeted in this paper is given in the Appendix, a subset of which is provided in Table 1 for the four fields that comprise our cosmology catalogue. We provide programme IDs for the community data used in Table 2.

The selected exposures were all processed through the DES singleepoch pipelines (Morganson et al. 2018) with the same configurations as those used for the DES Wide Survey (WS), and the griz DES SN observations were included in the global photometric calibration (see Burke et al. 2018). The photometric calibration for the remaining images was obtained by bootstrapping through a set of tertiary standards. The DES pipelines also assess the data quality of each observation which was used to select images for coaddition. The quality assessment yields for each exposure $i$ of duration $T_{i}$ an estimate of the PSF size $\mathrm{FWHM}_{i}$, the sky noise variance level $s_{i}$, and the atmospheric transmission $\eta_{i}$ relative to a clear night. From these we create an 'effective exposure time' (Neilsen et al. 2016),

$T_{\mathrm{eff}, \mathrm{i}} \propto T_{i} \frac{\eta_{i}^{2}}{s_{i} \times F H W M_{i}^{2}}$.

$T_{\text {eff,i }}$ is proportional to the $(\mathrm{S} / \mathrm{N})^{2}$ of a faint point source of fixed magnitude for that exposure. We normalize $T_{\text {eff }}$ for each band such that a value of unity is obtained for a typical $90 \mathrm{~s}$ DES WS exposure taken in clear, dark conditions.

\subsubsection{Selection of input images}

Each of the science goals for the DES Deep Fields is optimized by building the lowest-possible-noise image at or below a given target effective resolution (FWHM), or the smallest possible FWHM at a given effective noise level. To optimize the depth/resolution trade-off in a 
Table 1. DES Y3 Deep Fields position, area, $i$-band FWHM, exposure time and depth, number of sources detected in the COADD_TRUTH detection images (after applying masks) and number of chips excluded due to scattered light or other large-scale image quality defects. Exposure time, FWHM, and depth information for all pointings, depth levels, and DECam filters is given in Table A1.

\begin{tabular}{|c|c|c|c|c|c|c|c|c|}
\hline Field & $\begin{array}{c}\mathrm{RA} \\
(\mathrm{J} 2000)\end{array}$ & $\begin{array}{c}\text { Dec. } \\
(\mathrm{J} 2000)\end{array}$ & $\begin{array}{c}\text { Mask-free area } \\
\text { (sq. deg.) }\end{array}$ & $\begin{array}{l}\mathrm{FWHM}_{i} \\
(\operatorname{arcsec})\end{array}$ & $\begin{array}{l}\text { Exp. time } \\
\text { (s) }\end{array}$ & $\begin{array}{l}\text { Depth }_{i} \\
(10 \sigma, 2 \\
\operatorname{arcsec})\end{array}$ & $N$ sources & $N$ excl. chips \\
\hline $\mathrm{SN}-\mathrm{C} 3$ & $52.6484(03: 30: 35.6)$ & $-28.1000(-28: 06: 00.0)$ & 1.70 & 0.72 & 5862 & 25.06 & 462739 & 13 \\
\hline $\mathrm{SN}-\mathrm{X} 3$ & $36.4500(02: 25: 48.0)$ & $-4.6000(-04: 36: 00.0)$ & 1.52 & 0.77 & 4122 & 25.04 & 430555 & 16 \\
\hline SN-E2 & $9.5000(00: 38: 00.0)$ & $-43.9980(-43: 59: 52.8)$ & 1.42 & 0.83 & 5620 & 25.06 & 386946 & 17 \\
\hline COSMOS & $150.1166(10: 00: 28.0)$ & $+2.2058(+02: 12: 21.0)$ & 1.24 & 0.94 & 8885 & 25.54 & 403147 & 3 \\
\hline Total & - & - & 5.88 & - & 24489 & - & 1683387 & 49 \\
\hline
\end{tabular}

Table 2. DECam community programmes used in conjunction with DES imaging to construct the Deep-Fields images.

\begin{tabular}{lccc}
\hline Program & PI & Field & Bands \\
\hline 2014B-0613 & Cooke & SN-C3 & $u$ \\
2015B-0603 & Infante & SN-C3 & $Y$ \\
2016A-0620 & Curtin & SN-C3 & $u$ \\
2013A-0351 & Dey & COSMOS & ugrizY \\
2013A-0529 & Rich & COSMOS & $u$ \\
2014A-0073 & Kilic & COSMOS & $g$ \\
2014B-0146 & Sullivan & COSMOS & riz \\
2014B-0404 & Schlegel & COSMOS & $g z$ \\
2015A-0107 & Belardi & COSMOS & $g$ \\
2015A-0608 & Forster & COSMOS & gri \\
2016A-0104 & Sullivan & COSMOS & $g r i z$ \\
2016A-0386 & Malhotra & COSMOS & $z Y$ \\
2016A-0610 & Infante & COSMOS & $z$ \\
2014A-0239 & Sullivan & SN-E1 & $u$ \\
2014B-0146 & Sullivan & SN-E1 & $u$ \\
2016A-0104 & Sullivan & SN-E1 & $u$ \\
2014A-0191 & Hildebrandt & SN-E2 & $u$ \\
2016A-0104 & Sullivan & SN-E2 & $u$ \\
2016B-0260 & Curtin & SN-X3 & $u$ \\
\hline
\end{tabular}

given band, we order the candidate exposures for a given field and band by increasing FWHM. An optimally weighted sum of the first $N$ images in this ordered list will then achieve the minimal noise for its output FWHM, and the minimal FWHM for its noise level. We quantify the expected depth and resolution of such a coadd by

$T_{\text {eff }}(N)=\sum_{i=1}^{N} T_{\text {eff }, i}$

$\operatorname{FWHM}(N)=\frac{\sum_{i=1}^{N} T_{\mathrm{eff}, i} \mathrm{FWHM}_{i}}{T_{\mathrm{eff}}(N)}$.

Varying $N$ thus describes the best available trade-off between noise and resolution for each filter and band. Fig. 2 plots the available trade-off in $i$ band for all the $\mathrm{SN}$ fields.

For each of the $10 \mathrm{SN}$ fields we create 3 images in the griz bands that represent different choices in this depth/FWHM trade-off:

(i) The DEEPEST coadd is targeted at maximizing the detectability of faint sources. It includes all exposures until relaxing the FWHM cutoff no longer substantially increases depth. The resultant targeted deep-image FWHM's are 1.2, 1.0, 1.0, and $0.9 \operatorname{arcsec}$ in $g, r, i$, and $z$ bands, respectively. The DEEPEST coadds for the SN-C3 and SN-X3 fields are exceptionally deep in the red: in $z$ band, they are $\approx 3000 \times$ deeper than a typical single WS exposure ('SE depth'), summing $\approx 250000$ s of exposure time, and reaching 3.2 mag deeper than the DES WS coadds.

(ii) The COADD_TRUTH Deep Fields are intended to provide galaxy images for Balrog injection, and priors on the 'noiseless' distributions of properties of galaxies detectable in DES WS coadds. We target a depth $10 \times$ the expected WS coadd depth, i.e. $T_{\text {eff }}=$ 80 in units of typical SE depth, with typically 38 input images per coadd. But we place a higher priority that the deep-field FWHM be no worse than the median SE FWHM of 1.10, 0.96, 0.88, and 0.84 arcsec in griz bands, so some of the shallow SN fields do not attain COADD_TRUTH depth of $T_{\text {eff }}=80$ in gri bands.

(iii) The SE_TRUTH: coadds are intended to produce galaxy images for injection into the single exposures with very good seeing, i.e. those for which the COADD_TRUTH images have significantly higher FWHM than the target SE's. The input images to the SE_TRUTH Deep Fields are chosen to meet the most restrictive of two criteria: $T_{\text {eff }}=20$, and FWHM better than 90 per cent of all WS exposures. This places an upper limit of $0.93,0.82,0.77$, and 0.72 arcsec on the griz FWHM's of SE_TRUTH coadds. All of the SE_TRUTH Deep Fields can attain sufficient resolution with $T_{\text {eff }} \geq$ 10 except for SN-X2 $r$ band.

Example $g r z$ colour images for these three depth levels covering half of one of the chips in the SN-X3 field are shown in Fig. 3. The available data for the $\mathrm{SN}$-field $u$ and $Y$ bands, and for the COSMOS field, come from a handful of nights and do not represent as wide a range of seeing conditions as is available for the $\mathrm{SN}$-field griz data. As a consequence, for each of these we create just a single deep coadd, using nearly all of the available data. For the COSMOS field, this results in FWHM values of 0.94-1.20 arcsec for the ugrizY bands.

\subsubsection{Pipeline changes with respect to DES Y3 GOLD}

The Deep Fields required some modest configuration changes to the DES multi-epoch (coaddition) pipeline used for the Y3A2 reductions on which the DES Y3 GOLD sample is built (see Sevilla-Noarbe et al. 2021). The standard DES COADD tiles are defined as a fixed set across the sky with extent of $\sim 0.75^{\circ} \times 0.75^{\circ}$. For the Deep Fields, we defined new coaddition 'tiles' that are each slightly larger than a single CCD 18.41 arcmin $\times 9.64$ arcmin and centred at the median location of each CCD within the DECam focal plane for a given field. A summary of the changes in the processing follows.

First, we added constraints to more strictly enforce which images contributed to the resulting coadd image. In the nominal WS pipeline, all calibrated survey images that meet the survey quality cuts are included. For the SN Deep Fields the input images at griz-bands were restricted to come from the same CCD. At $u$ and $Y$ bands, 



Figure 1. Locations and layout of the DES Deep Fields, comprising 10 supernova survey pointings (SN-C, X, E, and S) and one pointing covering the COSMOS field. Filled rectangles represent the subset of the Deep Fields' DECam chips that were used in building our Deep-Fields catalogue for the DES Y3 cosmology analysis (see Section 3.1), colour coded by difference in $i$-band 10 $\sigma$ limiting magnitude relative to the maximum depth in that field. Green outlines show VISTA near-infrared coverage from VIDEO and UltraVISTA. Empty chips in SN-C3, SN-X3, SN-E2, and COSMOS indicated those that were excluded entirely in our catalogue due to scattered light or other large defects (see Section 2.3).

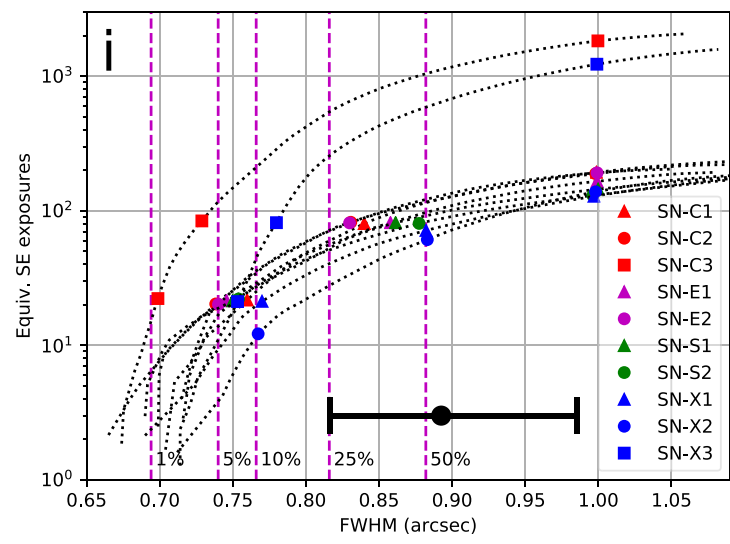

Figure 2. The dotted lines describe the depth-versus-resolution tradeoff available for the $i$ band in each of the 10 DES SN fields. The vertical axis gives depth in terms of effective exposure time, in units of a typical DES WS exposure. Dashed vertical lines mark the percentiles of FWHM for all WS single exposures in this band used in the Y3 data release. The black dot and error bar mark the median and 10-90 percentile range of seeing FWHM obtained in Y3 coadd images for this band. The coloured symbols mark the choices made in each field for depth/resolution of the three varieties of DeepFields coadd images. The three groups (from lower left to upper right) are SE_TRUTH, COADD_TRUTH, and DEEPEST. this restriction had to be lifted because the images were drawn from a much smaller population that included large offsets between the constituent exposures. For the COSMOS Deep Field, un-dithered data do not exist, however, many exposures exist that have telescope pointings with bore-sights within $\frac{1}{2}$ of a DECam CCD width/height of one-another. Thus, we loosened the restriction compared to that applied to the $\mathrm{SN}$ fields, and allowed adjacent CCDs to also contribute to a given COADD image. This procedure results in a more uniform depth across each image in the COSMOS field than we would have otherwise achieved.

Secondly, astrometric solutions are refit using all images in all bands simultaneously (using the AstrOmatic utility SCAMP, Bertin 2006). For the WS this function uses the objects from each exposure that overlap a given COADD tile. For the Deep Fields, only catalogues from the individual CCDs that overlap the tile are used. We used GAIA-DR1 (Gaia Collaboration2016), which was the best astrometric reference catalogue available at the time when this processing occurred, and ensure that our astrometric accuracy is at least as good as the WS $(\sim 150$ mas $)$.

In the Y3 DES COADD pipeline detection, images were formed by constructing a combined $r, i, z$-band image using SWarp with COMBINE_TYPE CHI_MEAN. For the Deep Fields this was found to produce less robust detection of faint objects in the presence of diffuse emission, therefore the configuration was altered to COMBINE_TYPE 

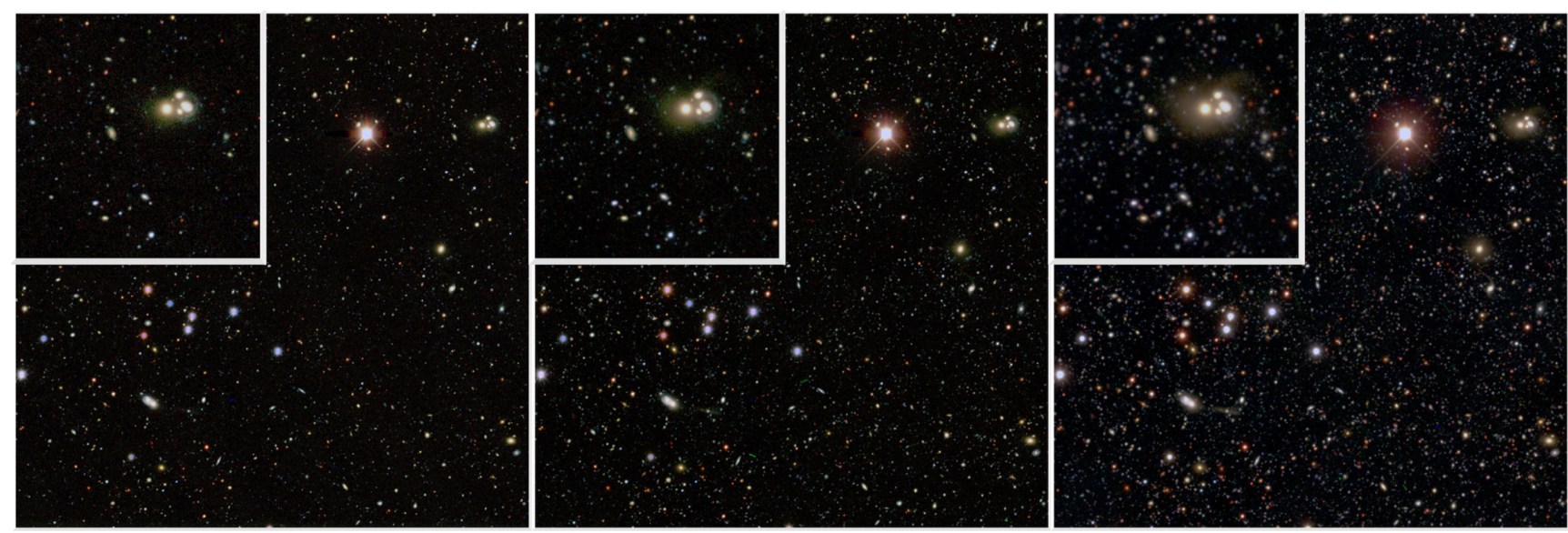

Figure 3. grz image of approximately one half of a DECam chip from the SN-X3 field, showing the three depth level coadd images we produce: SE_TRUTH, COADD_TRUTH, and DEEPEST, left to right, respectively. Inset figures are a factor 2 zoom-in of the interacting group located on the far right in the main image.

AVERAGE (i.e. a simple average of the images). Finally, alterations in object detection and PSF measurement are detailed in Sections 3 and 3.2 , respectively.

\subsubsection{Background subtraction tests}

Residual sky background estimation for the optical DECam images is performed using SExtractor, which can slightly overestimate the background due to the faint outskirts of some objects not being identified as source pixels. Once that background is subtracted, such an overestimation would lead to a small negative background when photometric measurement is performed. This is particularly important for our model-based photometry (see Section 3.3), as one of the components that is fit to the galaxy images follows a de Vaucouleurs profile, with $I \propto(1 / R)^{4}$. A poorly estimated background could therefore have a significant impact on the measured flux.

We check the accuracy of the sky subtraction by analysing the values of the pixels that remain after removing masked regions (see Section 2.3) and source pixels identified in SExtractor's segmentation check image. The distribution of the remaining pixel values should be approximately Gaussian and centred on zero, with the possible addition of a tail of high pixel values due to those source pixels that have not been included in the segmentation map and a low level of residual artifacts, which we can remove via clipping.

The left-most five panels of Fig. 4 show normalized histograms of pixel values (scaled to a common zero-point, $\mathrm{ZP}=30 \mathrm{AB}$ ) of all chips in the COSMOS area for each DECam band. Overlaid are bestfitting Gaussians, with mean $\mu$ and sigma $\sigma$ reported in the top right corners. In each case the pixel distribution is well represented by the Gaussian fit, and the mean value well within the standard deviation with respect to the zero (black solid line). Across these DECam chips, the background seems to be very slightly over subtracted, with mean values equivalent to surface brightnesses of 30.0, 29.3, 28.7, 28.2, and $27.6 \mathrm{mag} / \mathrm{sq}$. $\operatorname{arcsec}$ for $u, g, r, i$, and $z$, respectively. Our test does not probe any positional dependence of background over or under subtraction, and for simplicity we leave the minor offsets uncorrected in the following.

\subsection{Near-IR images}

The VISTA infrared camera (VIRCam; Dalton et al. 2006) on the VISTA telescope is a sparse array of 16 detectors within its field of view, arranged to allow a contiguous mosaic to be constructed from six pointings, covering $1.5 \times 1.23 \mathrm{sq}$. deg.. The VIDEO survey contains a total of eight such mosaics across three extragalactic fields: XMM, ELAIS, and CDFS. UltraVISTA comprises a single mosaic, but with half of the pointings taking almost seven eighths of the total survey exposure time, resulting in stripes that are $0.5-1$ mag deeper than the remaining three pointings. Further details of the VIDEO and UltraVISTA data are given in Jarvis et al. (2013) and McCracken et al. (2012), respectively, and point-source depths and mean seeing values are provided in Table 3 .

To build our near-infrared images we begin with all non-deprecated frame stacks in all broad-band filters ${ }^{3}$ taken as part of the VIDEO (Jarvis et al. 2013) and UltraVISTA (McCracken et al. 2012) public surveys that were available as part of the VIDEO and UltraVISTA DR4 release. Images are served via the Vista Science Archive ${ }^{4}$ following data reduction at the Cambridge Astronomical Survey Unit (CASU). The CASU reduction pipeline stages include corrections for detector reset, linearity, darks, flat-field and sky background. Images are further destriped and jitter stacked to account for bad pixels. These steps are described in detail in the CASU online documentation. ${ }^{5}$ The two surveys were carried out over 15 semesters between 2009 October and 2018 February (DR4 includes data up to and including semester 2014B), and contain 8987 (VIDEO) and 1485 (UltraVISTA) such frame stacks across all filters.

\subsubsection{Image processing and coaddition}

VISTA frame stacks come in the form of multi-extension Flexible Image Transport System (FITS) files, with one extension for each of the 16 chips in a single pointing. These files were unpacked into individual chip images, fluxes scaled to a common zero-point of 30 mag. using the image header zero-point and the noisy edges of each image were trimmed by setting the corresponding weight map to zero in the 100 pixel region around the border. In selecting the frames to go into our final coadded images, we chose to follow the quality assurance scheme used in the VIDEO survey and excluded only

\footnotetext{
${ }^{3}$ Our final catalogue contains just the set of $J, H$, and $K s$ bands that cover all of our selected fields.

${ }^{4}$ http://horus.roe.ac.uk/vsa/

${ }^{5}$ http://casu.ast.cam.ac.uk/surveys-projects/vista/technical/data-processing
} 

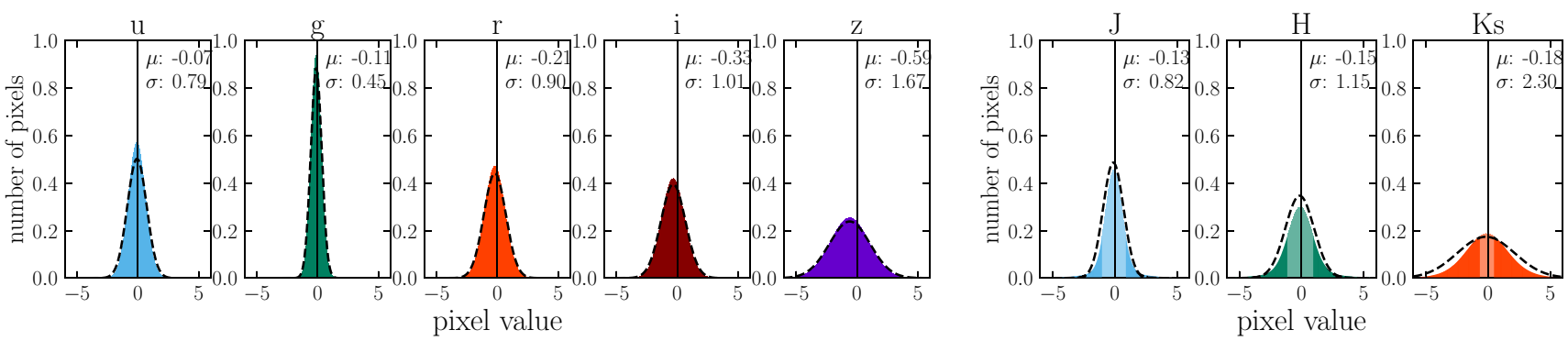

Figure 4. Histograms of pixel values for the optical DECam filters used in this work, after removal of pixels coincident with sources and masks. Here, we show a stack over all chips for each band, together with Gaussian function fits to the distribution. The background is very slightly oversubtracted: mean values correspond to surface brightnesses of 30.0, 29.3, 28.7, 28.2, 27.6, 29.3, 29.2, and $29.0 \mathrm{mag} / \mathrm{sq}$. $\operatorname{arcsec}$ for $u, g, r, i, z, J, H$, and $K s$, respectively.

Table 3. Median $10 \sigma$ depth in 2 arcsec apertures and PSF FWHM for the VISTA data used in this work.

\begin{tabular}{lcccc}
\hline Field & Programme & $J$ & $H$ & $K$ \\
\hline SN-C3 & VIDEO & $23.5,0.8 \operatorname{arcsec} 22.7,0.8 \operatorname{arcsec} 22.8,0.8 \operatorname{arcsec}$ \\
SN-X3 & VIDEO & $23.8,0.8 \operatorname{arcsec} 23.3,0.8 \operatorname{arcsec} 22.9,0.8 \operatorname{arcsec}$ \\
SN-E2 & VIDEO & $23.0,0.8 \operatorname{arcsec} 23.2,0.8 \operatorname{arcsec} 22.9,0.8 \operatorname{arcsec}$ \\
COSMOS & UVISTA* $^{*}$ & $24.0,0.77$ & $23.6,0.76$ & $24.0,0.78$ \\
& & $\operatorname{arcsec}$ & $\operatorname{arcsec}$ & $\operatorname{arcsec}$ \\
\hline
\end{tabular}

${ }^{a}$ Numbers refer to the deep, rather than ultradeep, stripes.

those frames that have reported seeing greater than 1 arcsec FWHM. Note that the release mosaics in the UltraVISTA survey were built from a lower level data product with stricter quality control and an improved treatment of the background subtraction. One of our chief concerns is producing a consistent set of photometry across our different fields, and so we treated the UltraVISTA frames in the exact same way as we did the VIDEO ones, rather than attempting to replicate their process.

Coadded images were produced for each combination of pointing and chip number. We chose to build distinct coadds for each pointing in order to keep the variation in PSF across each image as simple as possible, without sharp discontinuities at the joins of different chips and pointings. To build the coadded images we used Swarp with sigma_clipping $=3.5 \sigma$, i.e. input pixel rejection for values further than $3.5 \sigma$ from the mean value in that pixel.

The resulting images contain a number of cosmetic defects, including an electronic effect called detector column pull-down which has previously been observed in Spitzer data and the UKIDSS UDS. We followed Almaini et al. (in preparation) in correcting for this effect by median filtering the background, excluding source pixels identified using SExtractor, with a thin rectangular tophat kernel of dimensions $200 \times 3 \mathrm{pix}^{2}$. The filter is applied twice, with a transposed filter during the second pass. This process does a good job in correcting the visible stripes of background decrements and also corrects small amounts of oversubtraction that can occur around bright objects or moderately clustred regions of the images. However, it comes at the cost of further correlating the image noise, which is already correlated due to the SWarp resampling during coaddition. Any cosmetic issues that remain at this point were masked out (see Section 2.3).

\subsubsection{Background subtraction tests}

Although the background filtering algorithm that we employ has been tested in detail on the UKIDSS UDS (Almaini et al., in preparation), we nevertheless perform the same check that we applied to the optical data to measure the sky subtraction accuracy. We re-run SExtractor on the VIDEO and UltraVISTA single-chip coadd images, this time with background estimation fixed to a constant value of zero. The results are shown in the right-most three panels of Fig. 4 for the three near-IR bands. As expected, the median background level is extremely low in all three cases (29.3, 29.2, and $29.0 \mathrm{mag} / \mathrm{sq} \cdot \operatorname{arcsec}$ for $J, H$, and $K s$, respectively). This is by construction through the background correction process. The pixel distributions are clearly non-Gaussian, likely owing to depth variation from the dithered observations.

\subsubsection{Astrometry}

A shared astrometric alignment among the images in each band for each object is central to the multiband fitting algorithms used for galaxy photometry models. In the optical bands the DES pipelines accomplish this by simultaneously fitting astrometric solutions with SCAMP for all the images (in all bands) that contribute to a coadd tile. In order to achieve the same for the near-IR images, we used the DES catalogues as an astrometric reference and simultaneously fit all the constituent near-IR band images/catalogue to produce a bootstrapped astrometric solution for each near-IR image. We derived an alternative solution for the COSMOS/UltraVISTA field via a Gaussian Process regression of object positions using stars from the UltraVISTA DR3 catalogue (see Appendix A). This solution worked extremely well and was fully consistent with our SCAMP astrometry, providing further confidence.

\subsection{Image masking}

Because the deep coadds combine far more exposures than the WS coadds, there are many more opportunities for the coadd to become contaminated by unmasked single-exposure anomalies, particularly streaks from asteroids, meteors, and satellites. As a consequence, we implemented several improvements to the streak-detection algorithms to make the single-exposure masks more complete.

Masking was conducted on parallel but different tracks for the optical DECam and near-IR VIRCam images. In both optical and near-IR cases, we started with automated masks and supplemented them with an extensive manual masking campaign. For the DECam images, healsparse ${ }^{6}$ masks combine automated masks from the DESDM processing and manual masks into a single file, which are applied at the catalogue-level.

\footnotetext{
${ }^{6} \mathrm{https}: / /$ github.com/lsstdesc/healsparse
} 


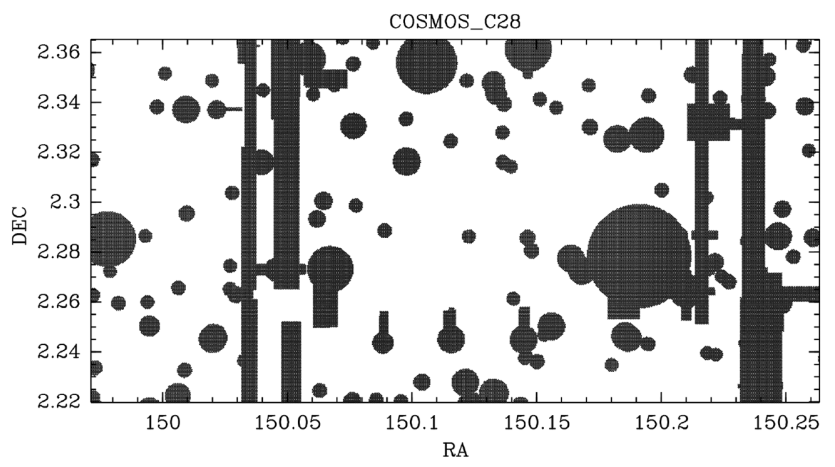

Figure 5. An example of masked regions in COSMOS, specifically on the C28 chip. This is an intersection of healsparse masks constructed for the DECam coadd and the UltraVISTA manual masks.

For the DECam images, the manual masking was performed by viewing colour images, so that artefacts were more easily identifiable. Typical artefacts and transients included cosmic rays, artificial satellites, meteors, and asteroids. DES 3-colour images of the Deep Fields were viewed on CCD-sized tiles by a team consisting of a mix of undergraduates and more experienced astronomers. Transient objects were flagged using a tool that recorded the center of the transient, the size of the circular patch that spanned it, and a comment about the shape of the transient, e.g. 'rainbow streak', 'single band point', 'weird thing', etc. A long streak could be covered by a sequence of small circular patches. Each tile was scanned several times. Images that were heavily contaminated by scattered light or any other large or extensive defects were identified at this point and later excluded during catalogue construction.

For the VIRCam images, we begin by constructing an automated mask around all infrared-bright stars, as follows:

(i) Using the Two Micron All Sky Survey (2MASS; Skrutskie et al. 2006a) Point Source Catalog (PSC), we identify RA and Dec. positions of point sources in $J, H$, and $K s$.

(ii) We then inspect a few randomly selected coadd images and manually apply circular regions to the point source positions using SAOImageDS9 (Joye \& Mandel 2003), masking out unusable areas of the images.

(iii) With these regions, we fit a second-order polynomial to obtain a function that determines the radius of a circular region given the magnitude of the point source.

(iv) Finally, we create masks for all near-IR images using this function.

Following the automated process, we then manually inspected all of the single-chip coadds, adjusting the base masks and applying additional polygons when necessary to areas affected by scattered light, stellar haloes, and other artefacts. Fig. 5 shows an example of the intersection of DECam and VIRCAM masked regions on a single chip in the COSMOS/UltraVISTA region, C28.

\subsection{Image depths and seeing}

Seeing FWHM, exposure time and $10 \sigma$ limiting depth ( 2 arcsec) for each DECam band, field and coadd depth level are provided in the Appendix, Table A1. In Fig. 1, we show the depth variation in the $i$ band for each field. Variations between chips of up to 0.3 mag can be seen in the SN fields, which are the result of a fraction of the input images being excluded from the final coadds due to cosmetic defects that were either not identified or were not able to be corrected for earlier in our pipeline. Meanwhile the effect of dithered pointings is clear in the COSMOS field. Here, the small gaps between chips and the field edges result in variations of up to 0.2 mag within individual chips, and a fall-off of depth at the field edges.

Depths and seeing FWHM for VIDEO and UltraVISTA are described at length in the relevant papers (McCracken et al. 2012; Jarvis et al. 2013). Our images are single-chip images built from the same input frames, and as such, match closely in depth and seeing. Average values for VIDEO and UltraVISTA are reported in Table 3, where the values given for UltraVISTA represent the shallower stripes (see Section 2.2 and McCracken et al. 2012).

\section{CATALOGUE EXTRACTION AND PHOTOMETRY}

We generate catalogues from the detection images of all depth level COADD images using SEXTRACTOR (Bertin \& Arnouts 1996). We use the same configuration as used for the DES Y3 processing (see Morganson et al. 2018), but with lower thresholds for detection and more aggressive deblending to accommodate the relative increase in objects in the Deep Fields compared to the DES main survey. The specific parameter changes used were: DETECT_THRESH 0.8 , ANALYSIS_THRESH 0.8, DEBLEND_MINCONT 0.00001, and DEBLEND_NTHRESH 64. The values of these parameters for the WS processing were arrived at by inspecting fields that include galaxy clusters or bright stars, and balancing the deblending and detection of true objects in the clusters against spurious sources near bright stars. In this work, we adjust them in order to better identify faint sources, as bright stars are later masked out. In the remainder of this section we detail the process of constructing the DES Deep-Fields catalogue for our Y3 cosmology analysis, beginning with the sub-selection of the fields we use.

\subsection{Field selection for the Y3 cosmology catalogue}

The scientific goals of the Deep Fields in part require the existence of longer wavelength near-infrared data to complement the optical DECam images, but also sufficient area to minimize sample variance uncertainties. We therefore choose a subset of the Deep Fields, with the aim of balancing the number of available photometric bands against the area of coverage. We select four pointings of the eleven we have at our disposal from which to build our main Deep-Fields catalogue: SN-C3, SN-X3, SN-E2, and the COSMOS field.

The SN-C3 and SN-X3 fields are the two deep DES SN fields which were also chosen to overlap with pre-existing spectroscopic data sets, such as the VVDS Deep 02hr field. All four fields substantially overlap with VISTA data, either from VIDEO or UltraVISTA, as can be seen in Fig. 1. The SN-X1 field is also largely covered by the VIDEO programme, and can be used to take advantage of spectroscopic redshifts from the UDSz (Bradshaw et al. 2013; McLure et al. 2013). However, at the time of data collection $J$-band images were not available for this pointing (VIDEO DR4). The field is also covered by the UKIDSS UDS, but the WFCam filters used for the UDS differ slightly from their VIRCam equivalents. For simplicity we do not use the SN-X1 field in building our catalogue, but intend to do so in future iterations.

As previously mentioned, one of the goals of the catalogue is to provide a list of objects that can be injected into the main DES survey area. This is in order to measure the transfer function relating noiseless true photometry to the photometry recovered by the detection and photometric measurement pipeline. The catalogue used for this purpose must be highly consistent in terms of filter responses 


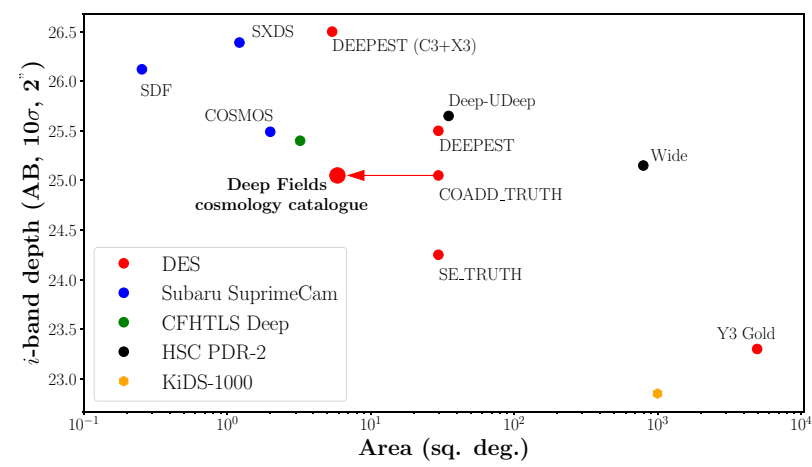

Figure 6. $i$-band depth (AB, 10, 2 arcsec) versus area for the DES Deep Fields and sub-selections relevant to this work, alongside a few other contemporary optical surveys.

and measurement pipelines, sufficiently deep as to be effectively noiseless, but also have high enough resolution in seeing FWHM to be able to reflect the characteristics of the main survey. These competing needs lead us to produce the COADD_TRUTH level of coadded images, and it is these that we use to construct our catalogue. The remainder of this paper concerns only these chosen images and the multiwavelength catalogue we build from them. A shortened summary of the characteristics of our selected fields is provided in Table 1, with fuller information provided in Table A1. The $i$-band depth and area of the images from which we draw our catalogue is shown in the context of other optical surveys in Fig. 6. These include the previously mentioned SXDS (Furusawa et al. 2008), COSMOS (Scoville et al. 2007), and HSC (Aihara et al. 2019), in addition to the Subaru Deep Field (SDF; Kashikawa et al. 2004), the Canada-France-Hawaii Telescope Legacy Survey Deep, ${ }^{7}$ and the Kilo-Degree Survey (KiDS; Kuijken et al. 2019). Note that KiDS has a similar wavelength coverage as described here, but includes a $Y$ band (for details, see Wright et al. 2019).

\subsection{Point spread function models}

We model the point spread function using a combination of SEXTRACTOR and PSFEx for both the optical and near-IR data, with some differences in the settings used for star selection that are detailed in the following.

\subsubsection{Optical PSFs}

The procedure is derived from the one used for the standard DESDM pipeline described in section 4.5 of Morganson et al. (2018). As an initial step, we run SExtractor on the coadds described in Section 2.1 with settings geared toward detection of point sources (DETECT_THRESH 5.0; DETECT_MINAREA 3) (cf. table 12 in Morganson et al. 2018). PSFEx is then run on the resulting SExtractor catalogue, which contains sub-images, or 'vignettes', corresponding to each detection. A key difference compared to the settings provided in table 13 of Morganson et al. (2018) is that when running PSFEx to automatically select stars, SAMPLE_MINSN ${ }^{8}$ is increased from 20 to 70. SAMPLE_MINSN controls the minimum

\footnotetext{
${ }^{7}$ https://www.cfht.hawaii.edu/Science/CFHTLS/T0007/CFHTLS_T0007-T echnicalDocumentation.pdf

${ }^{8}$ More information on PSFEx configurable parameters can be found at https: //psfex.readthedocs.io/_downloads/en/latest/pdf/.
}

$\mathrm{S} / \mathrm{N}$ of the vignette allowed into the star sample. Values of 30,50 , 70 , and 100 were all tested, and 70 was the value determined to best balance the goal of getting more than 100 PSF stars per DECam chip whilst not including galaxies, with the latter assessed by inspecting plots of FWHM (pixels) versus S/N. The default PSFVAR_DEGREES is the same as for the standard pipeline and is set to 2, so that the mapping of the PSF variations over pixel coordinates is done with a quadratic polynomial.

To validate the performance of the PSF models produced by PSFEX we investigated size residuals, where the size $T$ is given by

$T=I_{x x}+I_{y y}$

and $I_{x x}$ and $I_{y y}$ are second moments of the light distribution (Seitz \& Schneider 1997). For each of PSFEx's selected stars, we made cutouts of the image and weight files at the given location and fit the object using ngmix, ${ }^{9}$ a software package to fit Gaussian models to the light distribution, to obtain $T_{\mathrm{PSF}}$. We ingested the PSF model at the given location, drew it into a cutout using GALSIM (Rowe et al. 2015), and fit the object using ngmix to obtain $T_{\text {model }}$. A histogram of the median residual for each single-chip coadd $T_{\mathrm{PSF}}-T_{\text {model }}$ is shown in the left panels of Fig. 7 for COSMOS COADD_TRUTH, and fractional residuals $\left(T_{\mathrm{PSF}}-T_{\mathrm{model}}\right) / T_{\mathrm{PSF}}$ are shown in the righthand panel binned as a function of magnitude (MAG_AUTO) for the given filter. The data points indicate the fractional residuals averaged over all four fields fields shown, while the solid lines show the fractional residuals for each field for a sense of variation. These figures show that, overall, the PSF models meet the main stipulation of fractional residuals $<1$ per cent that is required to obtain accurate photometry measurements, shown as the shaded grey region.

\subsubsection{Near-IR PSFs}

For the near-IR data, we ran SEXTRACTOR with DETECT_THRESH 5.0 and DETECT_MINAREA 3 as before, although in this case background subtraction is turned off as it has already been subtracted during coadd image creation and processing. We then ran PSFEx to automatically select stars with SAMPLE_MINSN set to values ranging from 60 to 500 for UltraVISTA single-chip coadds and 70-100 for VIDEO single-chip coadds. These values were chosen after visual inspection of the stellar locus in plots of FWHM (pixels) versus $\mathrm{S} / \mathrm{N}$ that are automatically produced by PSFEX, and we revised the choice of SAMPLE_MINSN to account for different noise levels in some of the single-chip coadds. We imposed an additional criterion of $16<$ MAG_AUTO $<20$ and re-ran PSFEx on this selection of objects with PSFVAR_DEGREES set to 3 .

Histograms of the median residual for each single-chip coadd $T_{\mathrm{PSF}}$ $-T_{\text {model }}$ are shown in the left-hand panel of Fig. 8 (UltraVISTA, $\mathrm{SN}-\mathrm{C} 3, \mathrm{SN}-\mathrm{X} 3$, and SN-E2) with the three different colours representing the different filters $J, H$, or $K s$. Fractional residuals ( $T_{\mathrm{PSF}}-$ $\left.T_{\text {model }}\right) / T_{\mathrm{PSF}}$ are shown in the right-hand panel of Fig. 8 binned as a function of magnitude (MAG_AUTO) for the given filter. Comparison of the PSF residuals in the DECam optical bands (Fig. 7) and the VIRCAM NIR bands (Fig. 8) indicates that the PSFEx models are much more accurate for DECam, possibly owing to imperfections in non-linearity correction during the near-infrared data reduction.

\footnotetext{
${ }^{9} \mathrm{https}: / /$ github.com/esheldon/ngmix
} 

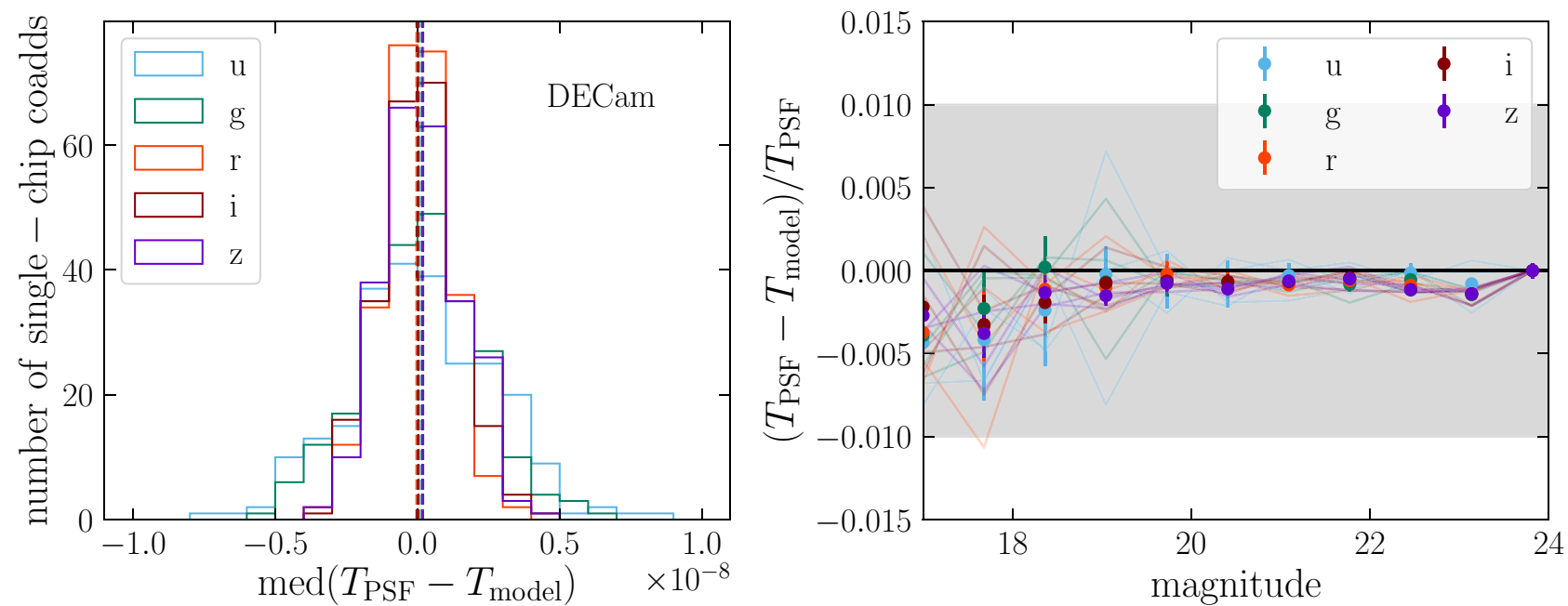

Figure 7. COSMOS and SN field PSF residuals. Left-hand panel: a histogram of the median PSF residuals for each single-chip coadd in the $u, g$, $r, i$, and $z$ filters. The median values of each histogram are plotted as dashed lines. Right-hand panel: Fractional residual PSFs binned by magnitude, which is taken here as MAG_AUTO in the given filter. Median values over all magnitude bins are plotted as solid lines (in this case not visible as they are so close to zero). The shaded grey band indicates a 1 per cent region. Solid lines are overlaid that indicate the spread of the four different fields, while the filled data points indicate the averages of all four fields.
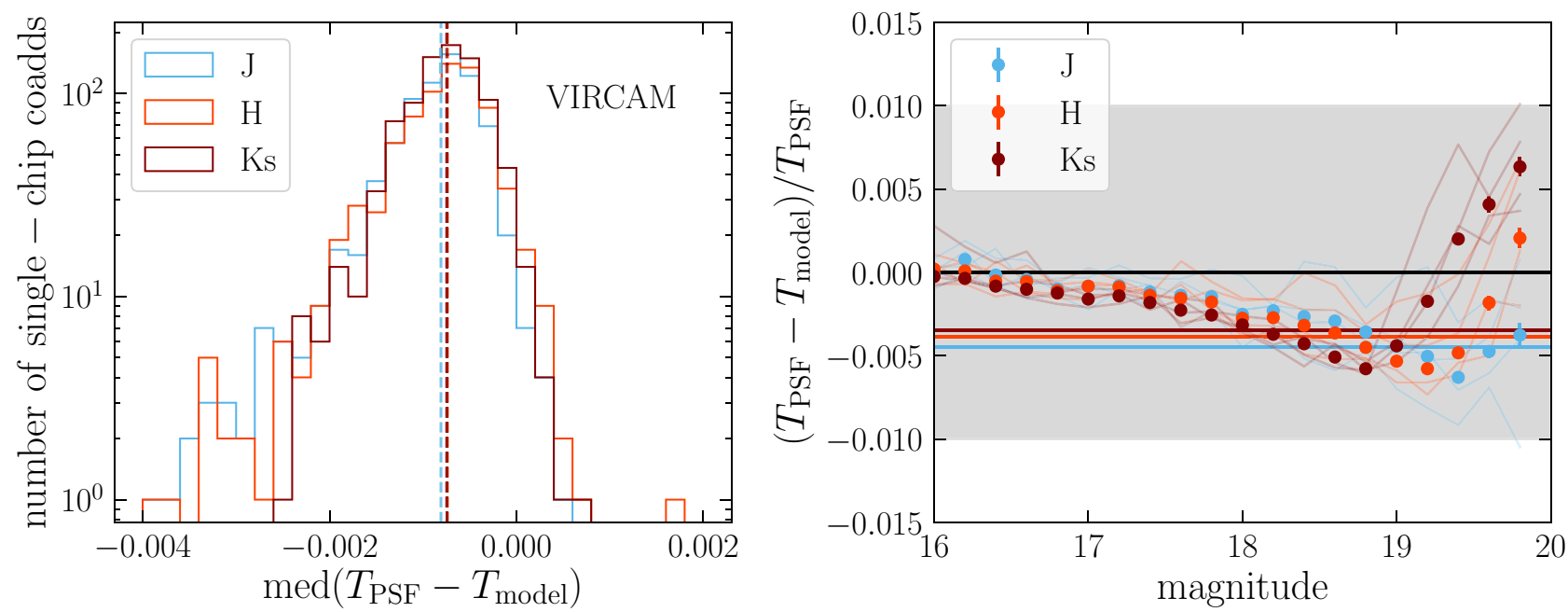

Figure 8. UltraVISTA and VIDEO PSF residuals, similar to Fig 7 but for UltraVISTA and VIDEO $J$, $H$, and $K s$ filters.

\subsection{Photometric measurement}

Our photometric measurements are based on a pipeline similar in spirit and approach to the Multi-Object Fitting (MOF; Drlica-Wagner et al. 2018) used to create Y3 GOLD but with some differences, necessary to handle the greater source density and addition of near-infrared data. The DES photometric pipeline is a development upon the ngmix shape measurement software for weak lensing measurements and was adopted chiefly to enable consistent galaxy model fits across a set of single-epoch images, rather than a single coadded image where the PSF may vary discontinuously across the image. In the case of the DES SN fields the single-epoch images are minimally dithered with respect to one another, and discontinuities in the PSF across a coadded image are therefore not a concern. Nevertheless, our key science goals require that the Deep Fields photometry is extracted in the same manner as the main survey (for the griz bands at least), and thus we use a pipeline built upon the same software principles, adjusting where necessary. The main steps to produce photometry measurements are:

(i) Object detection (see Section 2.1.1).

(ii) PSF measurement (see Section 3.2).

(iii) Reformatting input images into postage stamp image cutouts for fitting and collation of required metadata.

(iv) Formation of neighbouring object groups and deblending.

(v) PSF and galaxy model fitting, with simultaneous photometry measurement for the bands being fit.

(vi) Forced photometry measurements on the remaining bands.

We now describe the last four of those steps. 
The DES photometry pipeline is built around Multi Epoch Data Structures (MEDS; Jarvis et al. 2016; Zuntz et al. 2018), ${ }^{10}$ a fits data format developed to assist object-by-object operations, such as photometry and shape measurements, across large survey data. MEDS comprise the collated information for each object's single-epoch image cutouts, weight images, bit masks, segmentation footprints and associated metadata. One such file is produced per band and contains the information for all objects detected in a coadd riz detection image. The MEDS files are produced with a minimum cutout size of 32 pixels per side, up 256 pixels depending on the object's FLUX_RADIUS measurement from SEXTRACTOR (see Jarvis et al. 2016, Appendix A for details). The SExtractor-estimated background is also subtracted at this point, meaning that we are free to fix the background to zero during object fitting. MEDS files are produced automatically as part of the pipeline processing for our DECam data, but were built separately for the VISTA near-IR data.

We construct near-IR MEDS files based on the riz detection catalogue, using the optically determined RA and Dec. for each of the objects to make cutouts of the corresponding locations in the near-IR images and weights. Rather than use each individual frame for the near-IR, we use the coadded images as a single epoch. The individual exposures in the near-IR are shallow, due to the bright sky background, and many such exposures are required in order to build-up image depth. We consider the additional cost of processing so many epochs to far outweigh the possible gain in information retention. Note that the near-IR weight files have already had the masks described in Section 2.3 applied and thus contain zeros in any pixel that coincides with a mask. Furthermore, we save the corresponding PSF models described in Section 3.2 and segmentation maps that we generate with SExtractor. To create these segmentation maps, we run SExtractor over all the near-IR images and weights with -DETECT_THRESH 1.1, -ANALYSIS_THRESH 1.1, and -DEBLEND_MINCONT 0.001 . Finally, astrometric solutions from SCAMP, as described in Section 2.2.3 are converted into JSON format and saved in the MEDS files. We make no attempt to re-centre the near-IR image cutouts during photometric measurement, relying on the SCAMP astrometric solutions to be sufficiently accurate.

In a parallel operation to MEDS file construction, we form associations of neighbouring sources that may contaminate one another's measurements, and deblend them. For speed, this operation is performed on the coadded images with Shredder. ${ }^{11}$ The aim of the deblending at this stage is to form model representations of all the objects in a group simultaneously, using a set of Gaussian Mixture Models (GMMs). Each GMM representing a single galaxy consists of ten components, and the PSF is similarly represented by a fivecomponent model. Models are initialized as a de Vaucouleurs profile, but are free to evolve during fitting - only the centre is fixed.

Groups of objects are defined by forming friends-of-friends (FoF) groups of objects using the SExtractor segmentation image constructed from the DECam detection image, where a 'friend' link is defined as two objects having touching segmentation footprints. The vast majority ( $\sim 95$ per cent) of FoF groups in unmasked areas are singletons, and large groups are very few in number. Overall, $\sim 10$ per cent of unmasked galaxies are in groups of two or more members. This group forming stage, togther with GMM initialization based on SExtractor measurements, is performed with Shredx. ${ }^{12}$ All groups with two or more members are processed

\footnotetext{
${ }^{10}$ https://github.com/esheldon/meds

${ }^{11} \mathrm{https}$ ://github.com/esheldon/shredder

${ }^{12}$ https://github.com/esheldon/shredx
}

with the deblender and their resulting model fits are catalogued to be used during photometric measurement. Deblending objects with Shredder in this way has been found to work well even for object groups of up to 48 members (DES et al., in preparation).

\subsubsection{Model-fitting photometry}

For each object we return three sets of photometric measurements: PSF fluxes, bulge + disc model fluxes and Gaussian aperture fluxes, across all eight bands. Measurements are performed using the singleepoch images, with the exception of the near-IR images where the coadded images are used. When model fitting, the neighbours identified and deblended by Shredder are subtracted from the images using their catalogued GMMs.

PSF fluxes are a simple freely varying amplitude fit of the individual-epoch PSF models (described in Sections 3.2.1 and 3.2.2) at the position of the source in question, which is then integrated over the extent of the PSF model. In the case of the optical bands the final measurement is therefore a signal-to-noise weighted mean flux, while for the near-IR we have just a single effective epoch constructed from the coadded data. Our main photometric measurement is a bulge + disc galaxy model fit, described below. Finally, the Gaussian aperture fluxes that we produce are an analytical estimate, rather than a direct measurement, that applies a Gaussian-weighted aperture to an object's pre-seeing bulge + disc model, previously fit. These fluxes are robust against the possible noise or biases caused by the de Vaucouleurs component in bulge + disc measurements if the background is not perfectly estimated. They therefore enable important checks and comparisons during the Balrog process (Everett et al. 2020). The FWHM of the Gaussian aperture is fixed to 2.5 per cent.

\subsubsection{Bulge + Disc fluxes}

Our main flux measurements are derived from a bulge + disc model fit to the griz optical multiband, multi-epoch data, where the bulge and disc components are Sersic profiles with $n=4$ and $n=1$, respectively. The model is parametrized by the object centre (RA, Dec.), effective area (T), bulge-to-total ratio ('fracdev'), overall model flux for each band (griz) and the parameters governing the elliptical deformation matrix (g, which encode the position angle and axial ratio). The prior ranges for these parameters are given in Table 4. All of the individual epochs across the four griz optical bands are fit with the same model parameters for any given object. In the cases where an object is a member of a group, its neighbours are subtracted off before fitting, according to the model derived previously with Shredder. This model fitting step is performed by a new package, fitvd. ${ }^{13}$ However, it should be noted that, similar to MOF, fitvd is built on top of the core functionality of ngmix and thus they naturally produce highly consistent output measurements. One crucial difference between the two packages is the ability of fitvd to operate in a forced-photometry mode.

To enhance the stability of solutions and to reduce degeneracies in the parameter space, we restrict the freedom of the model space by fixing the relative effective radii of the bulge and disc components to be unity. We refer to the fluxes measured in this way as bulge + disc with fixed size ratio (BDF). While there are certainly galaxies for which this is a poor approximation, our main focus is on obtaining consistent and robust colours for the relatively faint galaxies that

\footnotetext{
${ }^{13}$ https://github.com/esheldon/fitvd
} 
Table 4. Prior ranges for the parameters used in our bulge + disc model fits.

\begin{tabular}{lll}
\hline Parameter & \multicolumn{1}{c}{ Prior type } & \multicolumn{1}{c}{ Prior range } \\
\hline$\delta$ RA & Gaussian & $\sigma=0.263 \operatorname{arcsec}$ \\
$\delta$ Dec. & Gaussian & $\sigma=0.263 \operatorname{arcsec}$ \\
Shape parameters, $\mathbf{g}$ & Bernstein \& Armstrong (2014b) & $\sigma=0.2$ \\
Effective area, $\mathrm{T}$ & Flat & $-1,1 \times 10^{5}$ \\
Fracdev & Truncated Gaussian & $\mu=0.5, \sigma=0.1, \min =0, \max =1$ \\
Flux & Flat & $-1000,1 \times 10^{9}$ \\
\hline
\end{tabular}

enter into the weak-lensing cosmology analysis, and where there is insufficient information to constrain a fully free bulge + disc model. Fitting was initialized based on the previously measured PSF fluxes and the second moments of the light distribution computed by SExtractor. Overall, only 1.26 per cent of unmasked objects result in a failed fit, with a bias towards objects in FoF groups with many members.

With final model fits in the main optical bands obtained, the final step is to perform the forced-photometry measurements on the remaining bands. fitvd takes the morphological parameters of the model that were determined from the griz bands, convolves the model with the appropriate PSF, renders it and fits the amplitude to the data. This forced-photometry mode was applied to the $u$-band and near-IR photometry. All members of an FoF group are fit at the same time.

\subsubsection{Similarities with alternative software solutions}

The ngmix software is not the only package that performs object fitting simultaneously across many input images for a large sample of objects. The most similar alternative software available is The Tractor (Lang, Hogg \& Mykytyn 2016). Both packages represent Sersic profiles and PSFs with combinations of Gaussians to allow fast convolution and share the same parametrization of object shapes (though with differing notation). The Tractor requires a driver script to operate on samples of more than a few to several objects. This script should make image cutouts around the objects of interest, handle the position-dependent PSF and initialize the fitting process. In our pipeline, building the MEDS format files performs an equivalent role, and so it is possible to adapt those files for use in The Tractor. We ran a small test area of one DECam chip to compare the photometry extraction via model fitting the four main DES bands, griz, and found excellent agreement.

Similarly, performing forced photometry on an image using a previously determined model for a galaxy or set of objects is not a novel concept. Perhaps the most commonly used code for this purpose in recent years is T-PHOT Merlin et al. (2015, 2016), notably in the deep survey areas of the CANDELS HST programme (Galametz et al. 2013; Guo et al. 2013). The use case for T-PHOT is often ensuring robust object colours from images with very different PSF sizes, e.g. combining HST images with ground-based imaging, or optical/near-IR images from 4 to 10 -m class telescopes with Spitzer data. In such cases the object footprints from the higher resolution image can be used directly, with a suitable convolution kernel to take account of the PSFs. In our case we do not have a band that is consistently of higher resolution (tighter PSF) than our other bands and so model fitting is the appropriate solution. T-PHOT can take the parameters of a model, such as the restricted bulge + disc model we use, and convolve it with the PSF to produce the object light profile to be fit against the data. Multiple objects can be fit simultaneously to deblend objects, and the intended functionality is therefore identical to our fitvd pipeline. Our decision not to employ one or both of The Tractor ${ }^{14}$ and T-PHOT is based on ease of pipeline implementation and consistency of code base with the main survey pipline.

\subsubsection{Variant for input to weak lensing image simulations}

We also constructed a special version of the photometry catalog for use in the Y3 weak lensing image simulations described in MacCrann et al. (2020). These simulations require realistic and well-constrained morphology as input to test the impact of blending on the shapes and photometric redshift distributions for the galaxies used for $\mathrm{Y} 3$ cosmology analysis. The input catalogue focuses on the COSMOS region and only contains photometry measured from the DECam griz bands. It is not simply a subset of the main catalogue described above because detections and fits to morphological parameters (halflight-radius, bulge-to-disc ratio, ellipticity) are based on the HST imaging, with bulge and disc components approximated by de Vaucouleurs and exponential profiles, respectively. These model fits are used to estimate fluxes in the DES griz filters from the DECam imaging described in Section 2.1. Fig. 3 of MacCrann et al. (2020) validates this catalogue by showing that the simulated distributions of quantities like magnitude, colour, $\mathrm{S} / \mathrm{N}$, and size match the observed quantities of the WS galaxies.

\subsection{Galactic reddening correction}

Interstellar extinction corrections to the ugriz photometry were applied following Schlafly \& Finkbeiner (2011) and described fully for the DES main survey GOLD sample in Sevilla-Noarbe et al. (2021). The procedure applied in the Deep Fields is the same as used for the fiducial correction in the DES main survey and we refer to that paper for details and associated uncertainties. Briefly, we compute the total extinction in a specific band $A_{b}$ for each object using the associated $E(B-V)_{\text {SFD }}$ reddening map value (SFD; Schlegel, Finkbeiner \& Davis 1998). To compute the $A_{b} / E(B-V)_{\mathrm{SFD}}$ coefficients in each band, we assumed a Fitzpatrick (1999) extinction law with $R_{V}=3.1$ and used the total transmission curves of each band for the calculation. For $J, H$, and $K s$ we use the coefficients from González-Fernández et al. (2018). Table 5 provides the $A_{b}$ coefficients for each band $b$.

\footnotetext{
${ }^{14}$ For completeness, we note that The Tractor can also operate in a forced-photometry mode.
} 
Table 5. Galactic reddening coefficients and magnitude zero-point (ZP) adjustments following field-to-field calibration and anchoring to the GOLD main survey sample. Adjustments are in the sense, $\operatorname{mag}_{\text {calib }}=\operatorname{mag}_{\text {meas }}$ + adjustment. Flux correction factors used during photo- $z$ estimation are also provided.

\begin{tabular}{lccccccc}
\hline Band & $A_{b}$ & SN-X3 & SN-C3 & SN-E2 & COSMOS & ZP uncertainty $^{a}$ & Photo- $z$ factor $^{b}$ \\
\hline$u$ & 3.963 & 0 & 0 & 0.034 & -0.03 & 0.055 & 1.105 \\
$g$ & 3.186 & 0.011 & 0.011 & 0.025 & 0.011 & 0.005 & 1.015 \\
$r$ & 2.140 & 0.006 & -0.004 & 0.02 & 0.006 & 0.005 & 0.977 \\
$i$ & 1.569 & 0 & 0.005 & 0.014 & 0 & 0.005 & 0.987 \\
$z$ & 1.196 & 0.004 & 0.004 & 0.019 & 0.004 & 0.005 & 0.993 \\
$J$ & 0.705 & 0 & 0.015 & 0.006 & -0.014 & 0.008 & 1.013 \\
$H$ & 0.441 & 0 & 0.015 & -0.002 & -0.004 & 0.008 & 1.025 \\
Ks & 0.308 & 0 & 0.015 & -0.002 & -0.004 & 0.008 & 0.984 \\
\hline
\end{tabular}

${ }^{a}$ Relative zero-point uncertainties.

${ }^{b}$ Multiplicative factors applied to catalogue fluxes during photometric redshift computation, derived from the fitting template spectra to PRIMUS galaxies (see Section 5.2.1).

\subsection{Photometric calibration}

The DES redshift methodology hinges on the consistency of the Deep-Field photometry across the different fields. This is especially true between the SN-VIDEO fields and the COSMOS-UltraVISTA field, as many of the key spectroscopic surveys are located in COSMOS but it is not covered by the main DES survey. The nearinfrared data are calibrated against the 2MASS survey (Skrutskie et al. 2006b) in all fields by the CASU reduction pipeline, though with relatively few stars per field available. Meanwhile, we could choose to tie the optical DECam data to the PanSTARRS survey (Chambers et al. 2016), but due to differences in filter transmission curves it is not clear that doing so would be an improvement over the tertiary standards that we used when building our images. We nevertheless compare our PSF photometry with that from HSC (Aihara et al. 2018), which is tied to PanSTARRS, in Appendix B.

Our approach for ensuring consistency between the four Deep Fields instead relies on matching stellar and galaxy loci between the fields, before tying to the DES WS using stellar sources. The remainder of this subsection details the following major steps:

(i) Compute expected differences in the colours of the stellar loci in our fields due to their sky position, using the Besançon Galaxy Model (BGM; Robin \& Creze 1986; Czekaj et al. 2014).

(ii) Compute median offsets in individual band and field combinations via the positions of stellar and red galaxy loci in a wide variety of colour-colour diagrams.

(iii) Perform an absolute calibration of the now relatively calibrated fields to the DES WS using stellar PSF photometry.

(iv) Estimate residual calibration uncertainties through comparing galaxy photometry from the Deep Fields and WS, and the residual scatter from the stellar and galaxy loci diagrams.

Final calibration adjustments and uncertainties are given in $\mathrm{Ta}$ ble 5 .

\subsubsection{Stellar locus matching}

Stars form a tight sequence in many colour-colour plots and in the absence of variations in population colours due to differing ages or metallicities, stellar sequences in different fields should lie on top of one another. Of particular use are combinations of colours where the stellar locus lies orthogonal to one of the axes, and extended along the other. In such cases we are able to perform a regression along the locus to check for offsets in colour between fields, and any colour dependence in such offsets as are found.

The observed population of Galactic stars in any given field depends upon the line of sight through the Milky Way, and in particular the relative contributions of bulge, disc and halo stars. Differences in metallicity (amongst other properties) at fixed stellar type result in small differences in the observed colours. If we assume that the stellar loci in our four fields are identical, and derive finetuned offsets to enforce this assumption, then we may actually introduce a systematic difference in the galaxy colours between one field and another.

The BGM is a four-component model of our galaxy, allowing an observer to compute the expected surface density, magnitudes, and colours of stars along any line of sight. We extract simulated stellar populations for each of our fields, using the web interface, ${ }^{15}$ and compute offsets in the position of the stellar locus in colour-colour plots of various combinations of our ugrizJHKs filters. We find no significant predicted offsets between the four fields ( $<0.2$ per cent), indicating that the stellar population (including metallicity) is similar across our fields. We now proceed to match the stellar loci in our observed data.

We perform a non-parametric regression by first estimating the density of stars in colour space through applying a kernel density estimate (KDE) to the population of stars in each field, using two colours at a time. We then identify the ridge-line of the sequence by searching for the maximum density and its associated value in one colour, conditioned on a value of the second colour in finely spaced intervals. That is, we find $\max \left(\operatorname{Dens}\left(c_{1}, c_{2}\right) \mid c_{2, i}\right)$ for $i \in\{1 \ldots k\}$, where $c_{2, i}$ are the finely sampled values of the second colour axis, numbering $k$ samples. We then compute residual differences for each field, relative to the mean of the four fields. Finally, a colour offset is determined though a median of the residuals over the useful range in $c_{2}$, determined by inspection of the fields' KDEs. The residuals are noisy due to intrinsic variation in the relative numbers of stars of different stellar types in each field, as well as shot noise and small photometric errors.

An example of one such colour-colour plot is shown in the upper half of Fig. 9. The four smaller panels in the upper right show the individual fields' KDE of their stellar population in the $g-r$ and $r-$ $i$ colours. The lower left panel shows that the populations do indeed

\footnotetext{
${ }^{15}$ https://model.obs-besancon.fr/
} 

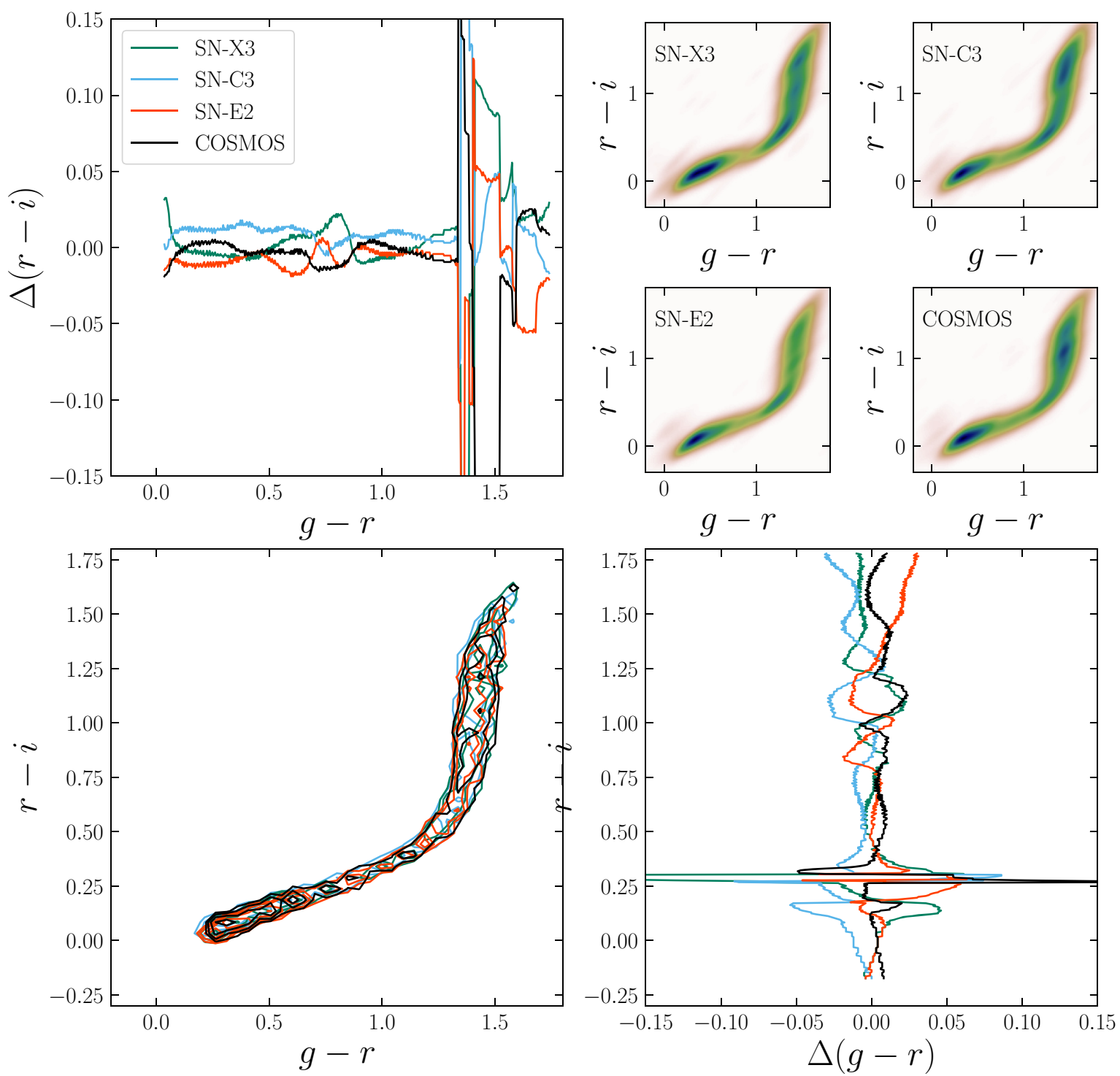

Figure 9. Example colour-colour residual diagnostic plot for stars in the $g-r, r-i$ colours space. In each set of four major panels we show 2D histograms in colour-colour space by way of number density contours (lower left) and a kernel density estimate (upper right), for each of the four fields. The remaining two panels show offsets of the location of maximum density for one of the colours, as a function of the other colour, versus the mean of the four fields.

lie on top of each other, as expected, while the remaining two panels show the residuals after subtracting the mean of the fields for the two colours.

\subsubsection{Red sequence galaxy locus matching}

Red sequence galaxies have very similar spectral energy distributions (SEDs) at fixed redshift, and a strong $4000 \AA$ break that allows them to be separated from the wider population, even in observed frame colours. For this reason, a red sequence galaxy sample drawn from a range in redshifts forms a tight sequence in some colourcolour combinations, and can be used in a similar way to the stars in a photometric regression. Over the area of each field, one or more square degrees, we expect general agreement in the location of the sequence in dereddened colour-colour space, with any sample variance entering as a source of noise.
We use the $g-z$ versus $z-K s$ colour space to select red sequence galaxies in analogy with the $B z K$ diagram (Daddi et al. 2004; Lane et al. 2007). The selection is shown in Fig. 10. In Fig. 11, we show the $i-z$ versus $g-K s$ colour space and residuals for our red sequence galaxy selection. Although far noisier than the equivalent figure for the stellar locus, it is clear that the $i-z$ colour is already very consistent across our four fields. Note that although there is a clear sample variance between the fields (particularly visible in the density plot for the SN-C3 field, top right of Fig. 9), it does not translate to a discrepancy in the colour of the locus.

We compute residual offsets with respect to the mean of the four fields for many combinations of colours for both the stellar locus and red galaxy sequence. Where we find a consistent need for a change in the zero-point across a few or more such diagrams, we compute it as the mean of the indicated offsets. Operationally, we begin by looking for consistent shifts in the $g, r, i$, and $z$ bands. Following that, 




Figure 10. Selection diagram for the red-sequence galaxy population used in fine-tuning the interfield calibration. Similar to a $B z K$ diagram (Daddi et al. 2004), the $g-z$ and $z-K$ colours cleanly separate the stellar locus from the galaxy population and allow the isolation of the red sequence at intermediate redshifts (Lane et al. 2007), shown by the purple dashed selection region. Galaxies in this sequence should have the same colours in different fields, and are used as part of our regression analysis.

we calibrate the near-IR bands, and finally the $u$ band. These small adjustments (typically $\leq 1$ per cent) are stored in table of band-field combinations and applied when computing differences with respect to the WS, which we come on to now.

\subsubsection{Calibration to DES Y3 GOLD}

The uniformity of the photometric calibration across the DES footprint is at a level $<0.3$ per cent (Sevilla-Noarbe et al. 2021), and roughly 1 per cent in absolute calibration (DES collaboration, in preparation). The $\mathrm{SN}$ fields overlap the main survey and so for bright sources the main survey data provide an excellent anchor. Moreover, a key target use of the Deep Fields is to provide source injections to the main survey, and so consistency between the Y3 WS GOLD sample of the main survey and the Deep-Fields catalogue is a firm requirement. We have already performed a photometric matching of our fields to one another in terms of their colours. The remaining freedom in calibration is therefore in the form of either coherent shifts across all four fields in a single band, or shifts in all the bands of a single field.

To perform the anchoring to the WS we position match the Deep-fields catalogue with stellar sources from the GOLD catalogue (Sevilla-Noarbe et al. 2021) with a tolerance of $0.5 \operatorname{arcsec}$ and then cut the sample to $18<i<20$ on the Deep-Fields PSF photometry. This cut is to reduce the impact of photometric errors and to ensure that any residual defects in background subtraction have minimal influence. Using PSF magnitudes for both the Deep Fields and WS, we then compute median offsets for each SN field against their WS counterparts and use the mean of these field-based offsets to perform the two calibration freedoms described above. Final photometric adjustments are reported in Table 5.

\subsubsection{Final zero-point offsets and uncertainties}

Using, for example, the mean of the $i$-band offsets computed for stellar sources in each field does not guarantee that they will all be perfectly aligned in terms of photometry to the GOLD sample. Any remaining differences can be used to quantify the accuracy of our calibration process, and to do so we repeat the above procedure but this time with galaxies. We allow a positional match within 1 arcsec to account for the greater centroid uncertainty in fainter, extended sources, and cut the resulting matched catalogue at $i<22.5$ in model magnitude. Histograms of bulge + disc model magnitude and colour differences for matched galaxies are shown in Fig. 12. Coloured steps represent individual fields, while the filled grey histogram shows the combination of the three SN fields. Source numbers are normalized to ease comparison of the fields.

The standard deviation of the median offsets between the Deep Fields and WS visible in Fig. 12 is the main contributor to our estimate of final zero-point uncertainty for the griz bands. In addition, we return to the stellar and galaxy loci diagrams shown in Sections 3.5.1 and 3.5.2, together with many different permutations of band combinations not shown in these figures. We used multiple diagrams to determine zero-point adjustments for each band, taking the mean of all usable such diagrams. The standard deviation from these measurements is therefore a second way in which we estimate the calibration uncertainty. For $u J H K s$ bands, these diagrams are our only source of information to estimate the residual zero-point uncertainty.

Individual band estimates of uncertainties are noisy, and so we group the bands into three sets for which the pipeline processing was homogeneous and combine their values: the main survey bands, VISTA bands and the $u$ band. Our estimated uncertainty in griz is 0.5 per cent, and if corrected for residual error in the wide field calibration, shows that the fitvd deep field version of the model fitting photometry works extremely well. For the VISTA bands we find a slightly larger error at 0.8 per cent, largely due to the fact that we account for the global optical - NIR error in the VISTA bands. The $u$ band was found to be rather more difficult to calibrate consistently across fields because the associated colour-colour plots are very noisy, resulting in a 5.5 per cent uncertainty that reflects our inability to estimate the error as much as the calibration itself. The final uncertainties in our photometric calibration are reported in Table 5, and as an additional check we compare our photometry with HSC Deep-UltraDeep photometry in Appendix B.

\subsection{Raw number counts}

Fig. 13 shows raw source number counts, uncorrected for incompleteness, as a function of $i$-band magnitude for our four Deep Fields (COADD_TRUTH depth level, colour stepped histograms) and the main WS GOLD sample (filled grey histogram). The peak of the Deep Fields' number counts is $\sim 1.25$ mag fainter than for the GOLD samples, as expected for images with ten times the exposure time. The four Deep Fields are highly consistent with one another at bright magnitudes, but begin to diverge somewhat at fainter magnitudes. In particular, the SN-E2 and COSMOS fields fall away from the reference power-law behaviour (lower panel of Fig. 13) at brighter magnitudes than the SN-C3 and SN-X3 fields. Moreover, the number counts at the faintest magnitudes are highest in the COSMOS field. This latter observation is again expected, due to depth variation across the COSMOS set of images. Overall, the exposure time in COSMOS is a little higher than the three SN fields, but the data are drawn from observations with different pointings, which leads to shallower regions caused by the gaps between the DECam chips and the outer regions of the field. The slight deficit in objects at $i \sim 23$ in SN-E2 and COSMOS may well be a result of source blending, as the PSF is significantly broader in these two fields $(\sim 0.9 \operatorname{arcsec}$ versus $\sim 0.7$ arcsec FWHM). 

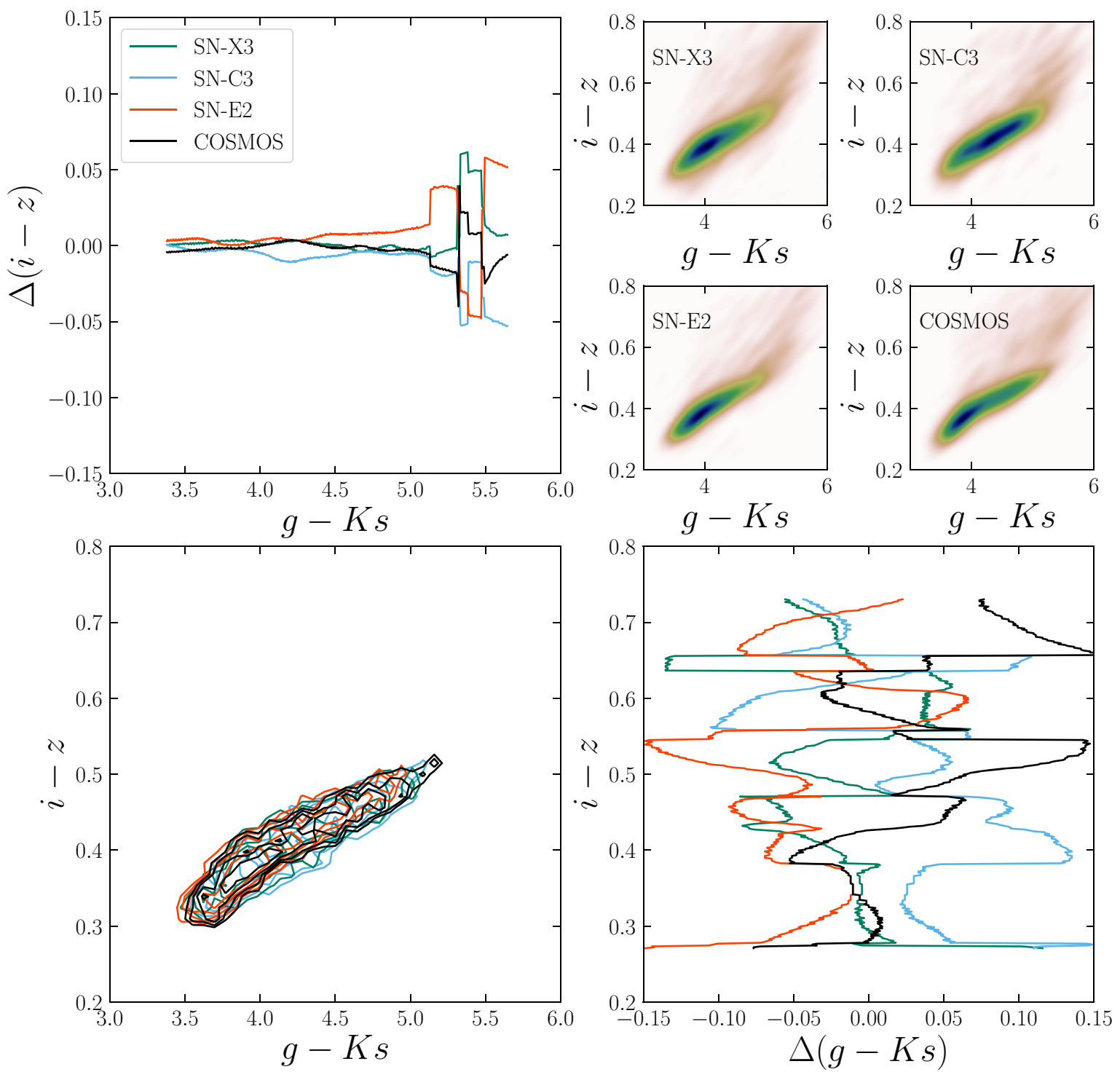

Figure 11. Same as Fig. 9, but for red sequence galaxies in the $g-K s, i-z$ space.

\subsection{Completeness}

In order to estimate source completeness for our cosmology catalogue (COADD_TRUTH level), we take advantage of the DEEPEST level of coadd images and the fact that our COSMOS field is largely overlapped by the UltraDeep level of HSC (Aihara et al. 2018). For the SN-C3 and SN-X3 fields, the COADD_TRUTH level of images contain just $\sim 6$ per cent of the $r, i$, and $z$-band data that make up the detection image of the DEEPEST coadds and is therefore effectively independent in noise realization. For the SN-E2 field, the fraction is 44 per cent, and thus the DEEPEST image in this field is not sufficiently deep nor independent enough for use in the following test. Given the similarity of the number counts in that field to SN-C3 and $\mathrm{SN}-\mathrm{X} 3$, however, we expect the magnitude-dependent source completeness will also be very similar.

The source lists from the deeper images, either HSC or our DEEPEST coadds, are treated as a truth table of objects that are present in the three fields. To this truth table we apply the set of masks defined for our Deep-Fields catalogue (including removal of CCDs in our ban list), described in Section 2.3. For SN-C3 and SN-X3, the masks remove most spurious sources and areas of scattered light, though a modest number of unidentified satellite trails and other contaminants may remain. We then perform a sky position match with a tolerance of 1 arcsec and compute the fraction of sources that are identified in our Deep-Fields catalogue, as a function of true $i$-band model magnitude. These fractions are shown in Fig. 14. A strength of this method is that our estimate includes all object morphologies that exist in the Universe and as such should be a better representation of the completeness than, for instance, a simulated point-source extraction.

For the two SN fields, the completeness curve matches very well the turn-over in number counts (Section 3.6) and shows our catalogue is highly complete ( $\sim 95$ per cent) at our reported $10 \sigma$ image depth, $i=25.05$. The interpretation of the COSMOS completeness curve is less straight forward. As mentioned in Section 3.6, the effective exposure time in the COSMOS $i$ - and $z$-bands is greater than for $\mathrm{SN}-\mathrm{C} 3$ or $\mathrm{SN}-\mathrm{X} 3$, leading to a larger number of very faint objects. 

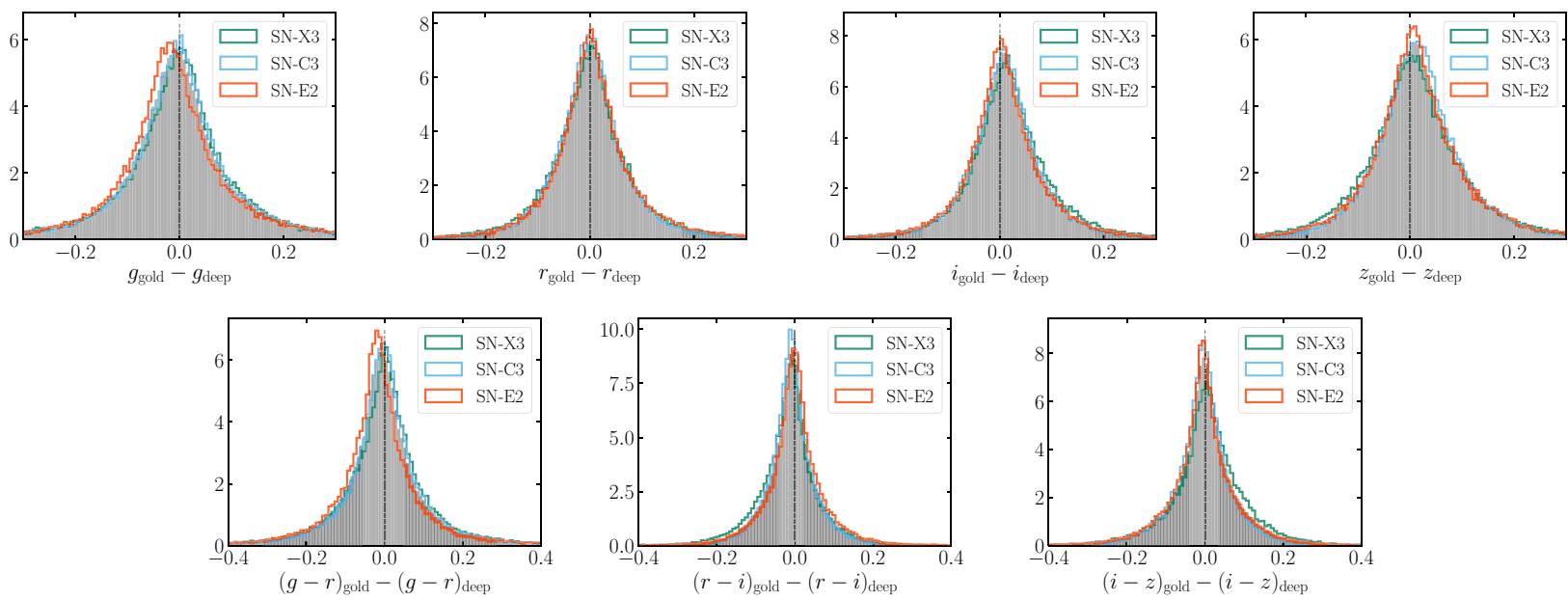

Figure 12. Comparison of the final Deep-Fields photometry with matched galaxies from the DES main survey Y3 GOLD catalogue, at $i<22.5$. Upper panels show single band magnitude differences, while the lower row of plots shows colours. Individual fields are as labelled, and the combination of all three SN fields is shown by the grey histogram. As the COSMOS field lies outside of the DES footprint it cannot be included in this figure. Histograms are normalized to emphasize differences in the median values.
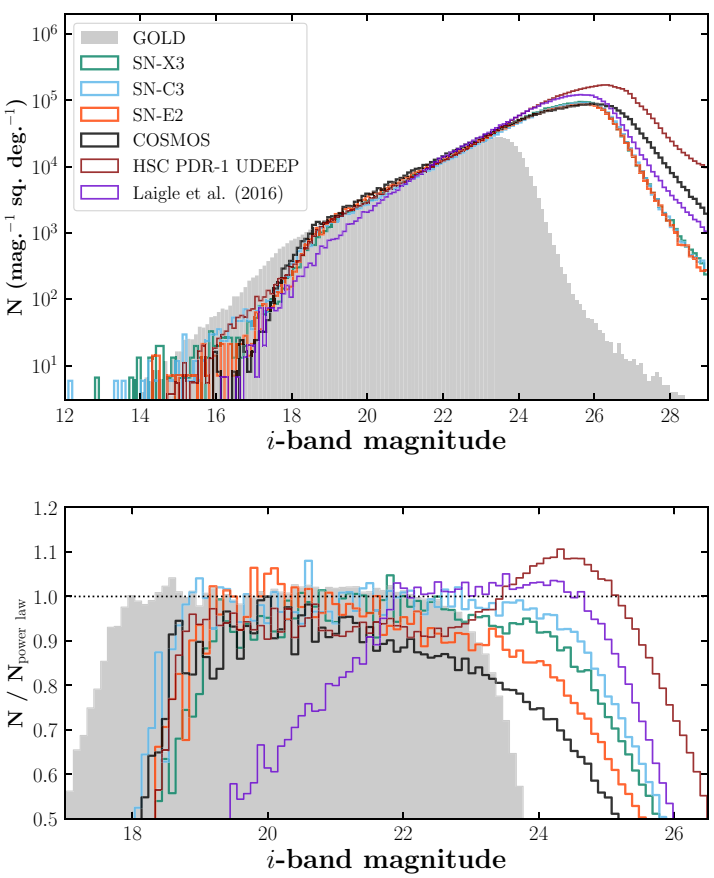

Figure 13. Upper: Raw number counts (uncorrected for incompleteness) in the $i$-band for our four Deep Fields (COADD_TRUTH depth level), together with the main DES survey, HSC PDR-1 UltraDeep (COSMOS + SXDS) and the Laigle et al. (2016) UltraVISTA/COSMOS catalogue. The fall off in number counts at the bright end with respect to the GOLD sample is largely due to masking of bright stars and saturation (for the Laigle et al. 2016 catalogue). Lower: Ratio of number counts to a fiducial power-law distribution.

However, the exposures were not all taken with a consistent pointing which leads to depth variation due to the gaps between chips. Moreover, the source list depends on choices made during source extraction such as the minimum extent in pixels an object must have, or flux ratio threshold to deblend merged objects. Therefore, when comparing the output from two different pipelines (DES and HSC)

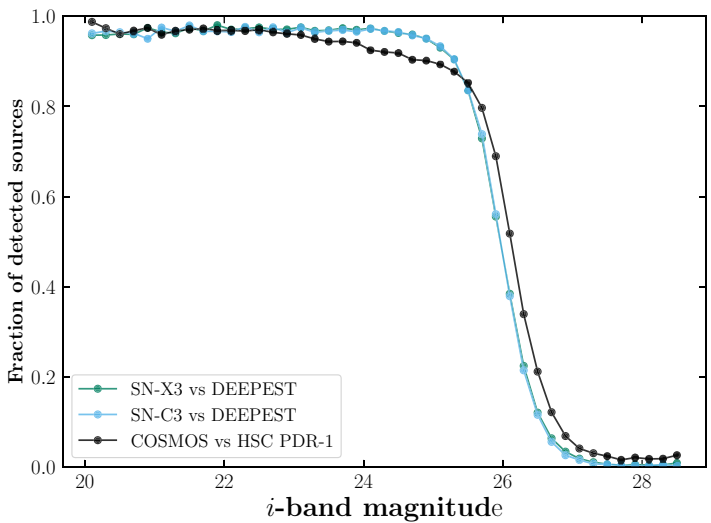

Figure 14. Detection completeness of sources in our Deep-Fields catalogue (COADD_TRUTH depth level) versus the DEEPEST level of coadd images (SN-X3, SN-C3) or HSC-PDR1 (COSMOS) as a function of magnitude. Deep-Fields masks were applied to the source lists, but some artefacts and spurious sources will remain in the DEEPEST and HSC data. The DEEPEST image in SN-E2 is not deep enough to produce a meaningful test for the COADD_TRUTH data. The data for SN-X3 and SN-C3 lie almost exactly on top of one another.

we are in part testing the response to those choices. Finally, the seeing FWHM in the HSC image is 0.62 arcsec, far superior to our COSMOS image at 0.94 arcsec. Even under the same extraction configuration, we expect a greater degree of source deblending from the better seeing image. Nevertheless, our reported completeness for the COSMOS field is still 90 per cent at $i=25$ th mag.

\section{STAR-GALAXY SEPARATION}

In order to carry out star-galaxy separation at faint magnitudes, we train a machine learning classifier using external data available on the COSMOS field. We choose to make this classifier independent of morphology and use only photometric information. We make use of all 8 bands, ugrizJHKs, and the Hubble Space Telescope Advanced Camera for Surveys (HST-ACS) observations of Leauthaud et al. (2007). From the HST-ACS catalogue we make use of the MU_CLASS 

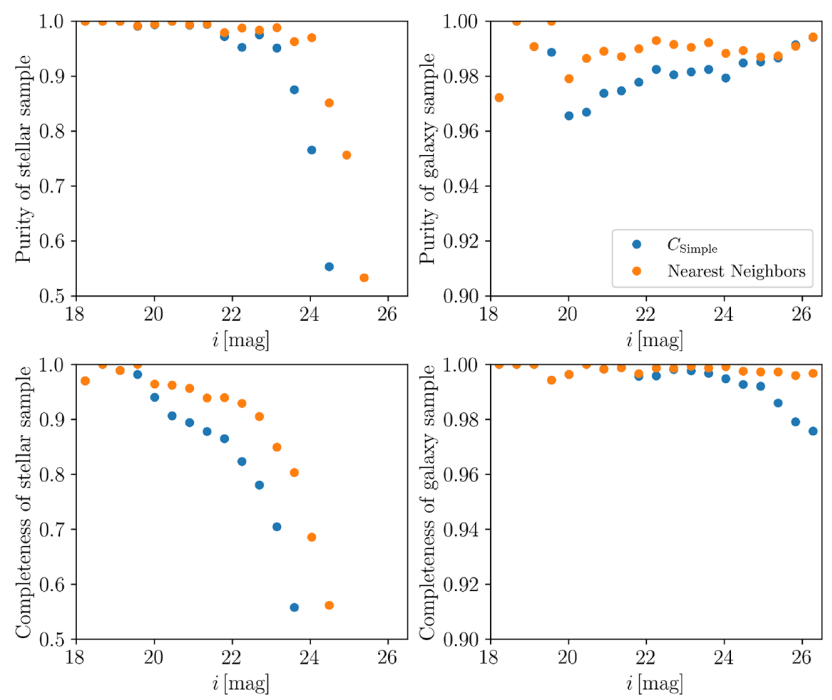

Figure 15. Purity (upper row) and completeness (lower row) for stars (left) and galaxies (right) for the machine learning classifiers considered, along with the colour classifier defined in equation (4). Numerical scores in the legend refer to the mean accuracy score.

star-galaxy classification as 'truth' labels. This classifier uses the high quality morphological information available from HST to identify a stellar locus in the surface brightness-magnitude plane.

We match the HST-ACS catalogue to the DES Deep-Fields catalogue and randomly select a sub-sample of 20 percent of objects to form a training set, ending up with 298338 objects in the training set. We then choose a number of supervised machine learning algorithms from SCIKIT-LEARN and use the MU_CLASS classifications as truth labels, and available colors in ugrizJHKs (colours formed from adjacent bands) as features. We find that a $\mathrm{k}$ Nearest Neighbors (kNN) classifier produces the best performance, as shown in Fig. 15. We also show the performance of a 'Simple colour class' classification, which takes the form:

$C_{\text {Simple }}=z-K-[1 / 3(u-r)+11 / 15]$

with galaxies having $C_{\text {Simple }} \geq 0$ and stars having $C_{\text {Simple }}<0$. Fig. 16 shows the resulting magnitude distributions and magnitudesize diagram for the different classes in the full catalogue. We subsequently apply the kNN classifier to the DES Deep-Fields data outside of the COSMOS field, in which HST-ACS MU_CLASS is not available, but ugrizJHK are.

\section{PHOTOMETRIC REDSHIFTS}

We employ the EAzY package (Brammer, van Dokkum \& Coppi 2008) to compute photometric redshift probability distribution functions (PDFs) for each object in the Deep-Fields catalogue. EAzY is a powerful and flexible template-fitting code, which uses linear combinations of a basis SED set derived through non-negative matrix factorization. The combination of SED components in this way allows a greater variety of galaxies to be modelled than can be achieved with the singular or pairwise interpolation SED fitting used in, for instance, BPZ (Benítez 2000). The costs to this approach include a longer runtime required per galaxy and the possibility of fitting to unphysical galaxy SEDs, although this latter concern can be somewhat mitigated by careful selection of the allowed combinations of base SEDs.
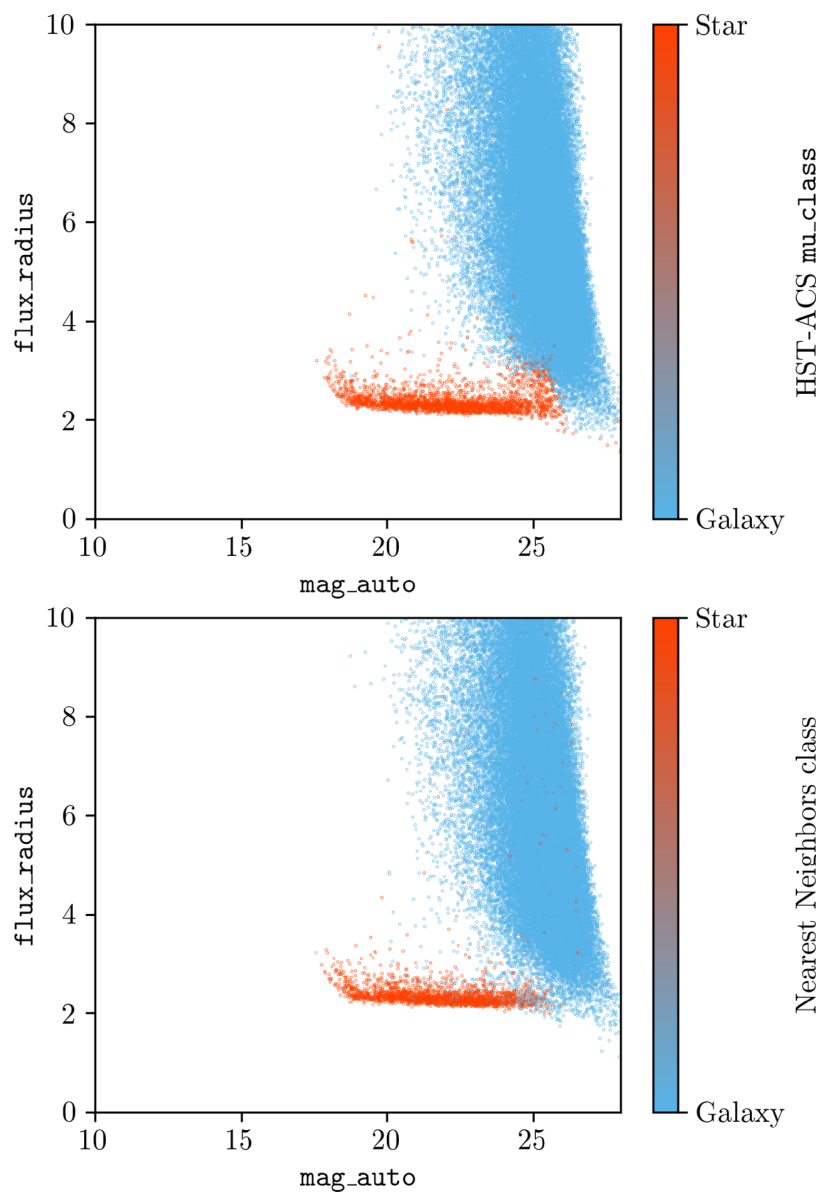

Figure 16. Size-magnitude diagram for the HST ACS morphological stargalaxy determination (upper panel) and kNN classifier used across all four Deep Fields.

The greater model freedom in the linear combination approach used by EAzY reduces the effective number of degrees of freedom in the $\chi^{2}$ computation, and leads to generally flatter likelihoods in redshift space. Moreover, the base SED components do not have explicit galaxy types (early type, late type, star-burst), and so cannot take advantage of our prior knowledge of how such types evolve in number with redshift. Instead, prior information is only included through apparent brightness. These factors make EAzY more suited to deriving redshifts for galaxies in richer fields, where high signal-tonoise photometric measurements are available across several bands or more (e.g. Hartley et al. 2013; Sherman et al. 2020). In such cases, we expect the redshift likelihoods to be fairly narrow and can use the greater model flexibility to improve precision and reduce outlier rate, relative to the single-SED case.

Our set-up with EAzY broadly follows the default configuration for the 12 Flexible Stellar Population Synthesis (FSPS) SED components. This template set was constructed using the method described in Brammer et al. (2008), but based on the FSPS stellar population synthesis model (Conroy \& Gunn 2010) and UltraVISTA photometric catalogue of Muzzin et al. (2013). We allow all possible combinations of components, include a systematic photometric error of 0.5 per cent motivated by Section 3.5.4 and include the default extended $R$-band-based prior. We soften the prior very slightly via a Gaussian convolution $(\sigma=0.1)$ on the high-redshift side of the peak of the prior. Our past experience with this prior has found 
that it penalizes high-redshift solutions a little too much (Euclid Collaboration et al. 2020), though this change is very minor. As the systematic uncertainty on the $u$-band zero-point is far larger than for the other bands, we include it in the object catalogue for the purposes of our photo- $z$ runs, added in quadrature with the computed photometric errors. We further perform a recalibration of the template error function (see Section 5.2.2) and allow redshifts up to $z=8$ in intervals of $\delta z=0.01$.

\subsection{Spectroscopy in COSMOS and SN fields}

The performance of photo- $z$ is typically tested against a subset for which true redshift values are available, in the form of high-confidence spectroscopic determinations. Such samples are inevitably biased towards brighter objects and those with clear spectral features that lie within the wavelength range of the spectrograph. Often, the chosen performance metrics are computed with a weight for each object in order to compensate for this bias and obtain results that better reflect a flux-limited selection of the photometric data set (Sánchez et al. 2014; Bonnett et al. 2016). Increasingly, very precise photo- $z$ from surveys of many medium and/or narrowbands are being utilized for this purpose also (Bonnett et al. 2016; Hoyle et al. 2018; Tanaka et al. 2018; Alarcon et al. 2020; Euclid Collaboration 2020). The advantage of using photo- $z$ is that they are by construction complete, and so don't suffer from the sort of selection biases discussed in Hartley et al. (2020). On the other hand, a fraction of the photo-z are likely to be wrong, with increasing prevalence at fainter magnitudes. Between these two extremes are very low-resolution grism or prism spectra, such as those in PRIMUS (Cool et al. 2013) and 3D-HST (Momcheva et al. 2016). These can be thought of as suffering the weaknesses of both spectroscopy and photo- $z$, but to generally much lesser degrees. In this work, we use these low-resolution samples to calibrate our method rather than test final performance.

In the three SN fields, SN-C3, SN-X3, and SN-E2, we use the spectroscopic compilation put together by OzDES, a partner survey of DES with the central goal to obtain SNe host galaxy redshifts. We wish to avoid biasing our performance measurements by an overabundance of galaxies with active nuclei (AGNs), which we know are not correctly modelled by the galaxy templates we employ. We therefore remove sources of spectroscopy that specifically targeted quasars or AGNs, and further remove AGN hosts where they have been identified in the spectroscopy metadata (e.g. via observer notes or specific quality flags). The sources of spectroscopy that we retain are listed in Table 6 together with the quality cuts we make. We also list the spectroscopic data sets that we use in the COSMOS field. As the best-studied extragalactic field, the spectroscopy in the COSMOS field is invaluable and is easily the most abundant of our Deep-Fields. We further use photo- $z$ from the COSMOS + UltraVISTA catalogue of Laigle et al. (2016) based on 30 photometric bands, including 12 medium bands from Subaru, and the recent addition of the 40 narrowband survey, Physics of the Accelerating Universe Survey (PAUS), in the same field, with redshifts determined by Alarcon et al. (2020).

\subsection{Flux recovery and model error budget}

Not all of the spectroscopic data sets are suitable for assessing the performance of photo-z measurements. For instance, the PRIMUS data include a non-negligible fraction of incorrect redshifts, even at highest confidence, due to the nature and difficulty of reducing and analysing prism spectra. Furthermore, redshift solutions for galaxy templates above $z=1.2$ were not included in the analysis pipeline
(Cool et al. 2013). Nevertheless, such a large sample of galaxies (13979, after applying cuts) where the vast majority of redshifts are indeed correct can still be of great use in calibrating zero-point corrections and a template error function, especially as the survey data include parts of all four of our Deep Fields.

\subsubsection{Zero-point calibration}

We test and calibrate the photometric zero-points for photo- $z$ determination through running EAzY with the redshift of each PRIMUS object fixed to its spectroscopic redshift. We use only objects with the most secure redshifts, that are classified as galaxies, are unflagged in our Deep-Fields catalogue and pass an $i<21.5$ cut. These cuts reduce the impact of photometric errors and colour-dependent incompleteness. The PRIMUS catalogue is 80 per cent complete under these cuts, however it remains possible that the level of incompleteness we allow could result in subtle biases (see Hartley et al. 2020). A separate run of EAzY is used for each of the eight photometric bands. In each run the photometric errors of the band in question are multiplied by a factor of $10^{15}$ so that the band does not contribute to the determination of the model SED coefficients. The fractional flux difference between the catalogue flux and the predicted flux from the best-fitting template combination, $f_{\text {cat }}-f_{\text {model }} / f_{\text {model }}$, is computed for each object. These fractional errors are shown against spectroscopic redshift in Fig. 17.

As noted earlier, a fraction of the redshifts will be incorrect, despite the fact that we use only the highest confidence objects. However, in using the median value within a running boxcar filter over redshift (width, $\Delta_{z}=0.2$ ), the influence of these wrongly assigned redshifts will be minimal. The results from the smoothing filter are shown by the red lines in Fig. 17. Deviations from unity in these lines can be caused by a number of issues, e.g. incorrect templates, errors in filter curves or incorrect zero-point calibration. Mis-calibrated photometric zero-points will appear as a redshift-independent offset, while discrepancies between the true and model SEDs will typically show up by deviations that are quite isolated in redshift, and will be seen with a similar form but at different redshifts for different bands.

While we see evidence for a benefit in applying some small zeropoint offsets, we see little evidence for the sort of clear fluctuations in flux difference between catalogue and model that would suggest an obvious problem with the template SED set. Though there are some notable features and redshift-dependent fluctuations, they are not coherent in rest wavelength between bands. The flux differences found for the $u$-band, and to a lesser extent the $g$-band, are broadly expected, given the great variance in UV spectra caused by relatively small changes in star-formation rate and internal dust extinction. This region of the spectral range has a large template uncertainty (see next subsection) in order to account for such issues. The $K s$ band panel also stands out, having an apparent redshift-dependent slope to the model discrepancies. We could interpret this behaviour as residual imperfect background subtraction, SED miscalibration at long wavelengths, or perhaps a problem with the filter response curve. Another possibility is that it could be due in part to the fact that we do not have a longer wavelength band to bracket the $K s$ band with, and the $H$ band is one where the zero-point seems to require additional calibration.

Using the results shown in Fig. 17 we derive photometric zeropoint corrections for the purpose of computing photo-z with EAzY. For each band, an offset is computed as the median value of the boxcar-filtered data (i.e. median value of the fractional flux difference displayed by the red lines). In this way, our calibrations are not biased 
Table 6. Sources of spectroscopic data used in photo-z performance metric assessment.

\begin{tabular}{|c|c|c|c|c|}
\hline Data set & Number & Fields & Flags $^{a}$ & Reference \\
\hline 2dFGRS & 297 & SN-C3,SN-E2 & $\geq 3$ & Colless et al. (2001) \\
\hline $2 \mathrm{dF}$ archive & 5494 & $\mathrm{SN}-\mathrm{C} 3, \mathrm{SN}-\mathrm{X} 3$ & 4 & - \\
\hline $3 \mathrm{D}-H S T$ & 6937 & SN-C3,COSMOS & - & Momcheva et al. (2016) \\
\hline $6 \mathrm{dF}$ & 48 & SN-C3,SN-X3,SN-E2 & 4 & Jones et al. (2009) \\
\hline ACES & 5479 & $\mathrm{SN}-\mathrm{C} 3$ & 3,4 & Cooper et al. (2012) \\
\hline $\mathrm{C} 3 \mathrm{R} 2$ & 2248 & COSMOS & $\geq 3.2$ & Masters et al. (2017) \\
\hline DEIMOS $10 \mathrm{~K}$ & 5931 & COSMOS & 3.x,4.x,23.x,24.x & Hasinger et al. (2018) \\
\hline FMOS COSMOS & 239 & COSMOS & 4 & Silverman et al. (2015) \\
\hline GAMA & 2363 & $\mathrm{SN}-\mathrm{X} 3$ & 4 & Baldry et al. (2018) \\
\hline GCLASS & 134 & SN-E2 & 1 & Muzzin et al. (2012) \\
\hline KMOS-3D & 326 & SN-C3,COSMOS & 0 & Wisnioski et al. (2019) \\
\hline LEGA-C & 1405 & COSMOS & 4 & Straatman et al. (2018) \\
\hline MOSDEF & 397 & SN-C3,COSMOS & 7 & Kriek et al. (2015) \\
\hline MUSE & 494 & $\mathrm{SN}-\mathrm{C} 3$ & 3 & Herenz et al. (2017) \\
\hline OzDES & 3924 & SN-C3,SN-X3,SN-E2 & 4 & Lidman et al. (2020) \\
\hline PanSTARRS & 412 & $\mathrm{SN}-\mathrm{X} 3$ & 4 & Rest et al. (2014) \\
\hline PRIMUS & 13979 & SN-C3,SN-X3,SN-E2,COSMOS & 4 & Cool et al. (2013) \\
\hline SDSS & 2325 & $\mathrm{SN}-\mathrm{X} 3$ & 0 & Abolfathi et al. (2018) \\
\hline SNLS & 401 & $\mathrm{SN}-\mathrm{X} 3$ & 1 & Bazin et al. (2011) \\
\hline VIPERS & 3493 & $\mathrm{SN}-\mathrm{X} 3$ & 3.x,4.x,23.x,24.x & Garilli et al. (2014) \\
\hline VUDS & 145 & $\mathrm{SN}-\mathrm{C} 3$ & 3.x,4.x,23.x,24.x & Tasca et al. (2017) \\
\hline VVDS & 3808 & $\mathrm{SN}-\mathrm{X} 3$ & 3.x,4.x,23.x,24.x & Le Fèvre et al. (2013) \\
\hline zCOSMOS & 12733 & COSMOS & 3.x,4.x,23.x,24.x & Lilly et al. (2009) \\
\hline
\end{tabular}

${ }^{a}$ Values of spectroscopic redshift quality used, as defined by the survey's quality flagging system.

towards the redshifts most populated by our spectroscopic sample. These zero-point adjustments are then applied to a new run of EAzY, again with redshift fixed to the spectroscopic redshifts, and the above procedure repeated. We iterate this process until stability, where the new zero-point adjustments across $r$ to $K s$-band are at the level of 0.5 per cent of flux or smaller. ${ }^{16}$ The final zero-point calibrations derived in this way are given in Table 5. Most adjustments correspond to $1-3$ per cent of the object flux, with the $u$-band being the clear outlier. It is worth noting that the difference in zero-point calibration between the $H$ and $K s$ bands is similar to that found by Laigle et al. (2016), which is perhaps unsurprising given the data in common and our use of the UltraVISTA SED set. The zero-point calibration difference between $J$ and $K s$-bands in the COSMOS field is also reasonably close to that in Laigle et al. (2016), once our earlier field-to-field calibrations are taken into account (Section 3.5.4).

\subsubsection{Template error function calibration}

The variance of real galaxy SEDs is difficult, if not impossible, to fully capture with a limited template set - even through a linear combination of 12 base SEDs. The equivalent widths of emission lines vary with redshift and also at fixed redshift and constant restframe colour, subtly altering even broad-band fluxes. Furthermore, intergalactic absorption affecting the rest-frame UV spectral range is a stochastic process that would take a great many SED components

\footnotetext{
${ }^{16} \mathrm{Because}$ the $u$ - and $g$-band probe the rest-frame UV the iterative process
} can be unstable, fitting less dusty SEDs with each iteration. to model. For these reasons (amongst others), Brammer et al. (2008) introduced a template error function to the EAzY package to capture any wavelength-dependent uncertainty or mis-calibration in the templates. The template error function is clearly dependent on the set of SED components under consideration, and included with $\mathrm{EAzY}$ is an error function appropriate for the UltraVISTA-derived set that we use. Nevertheless, its derivation is also weakly dependent on the photometric data used, and so we assess its performance with our Deep-Fields catalogue.

We follow the procedure described in Brammer et al. (2008), but use the same fractional flux differences measured from the PRIMUS cross-matched data as earlier in this section, fixing the redshifts at their spectroscopic values. Due to the fact that we expect some outliers arising from wrong spectroscopic redshift assignments, we apply a $5 \sigma$-clipping to the fractional flux differences, centred on the median and iterated five times, before computing their variance. This is again performed in a sliding window of $\Delta z=0.2$. The result of this process is shown by the short coloured lines in Fig. 18, representing the interval $0.1<z<0.8$ where we have a sufficient number of objects, and with the contribution to the variance coming from the typical photometric uncertainty subtracted out. Clearly, from around $5000 \AA$ onwards the template error function is already well matched to our data, with perhaps a small over estimate at the longest wavelengths. However, throughout the wavelength range probed by the $g$ and $u$ bands it is too small.

It is worth noting that, while the $g$ and $r$ bands agree well in their prediction for the required template error at $\sim 4000 \AA$, the $g$ and $u$ bands are highly discrepant. In Section 3.5.4, we estimated a systematic zero-point error for the $u$ band of 5.5 per cent. Interpreted 



Figure 17. Fractional flux offsets between measured galaxy photometry and those predicted through EAzY template fits at their spectroscopic redshift. The red solid line shows the median value within a sliding window of width $\Delta z=$ 0.2 and the black dashed line shows our computed zero-point correction.


Figure 17. - continued

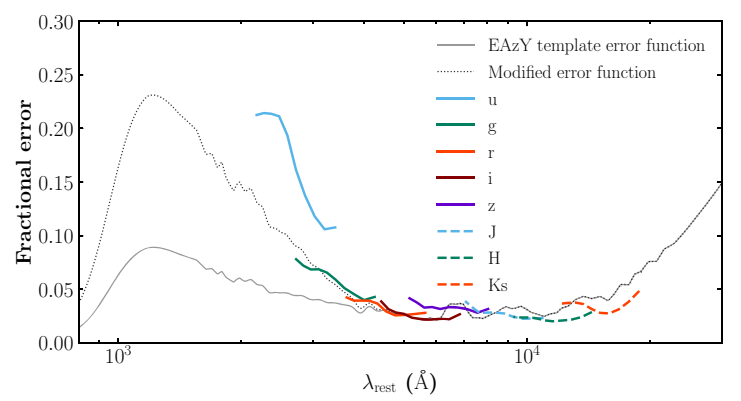

Figure 18. Dispersion in the measurements shown in Fig. 17 as a function of rest-frame wavelength, over the redshift range $0.1<z<0.8$. Coloured solid and dashed lines show the results for different photometric bands. The solid black line shows the default EAzY template error function for our SED component set, while the dotted line shows our adjusted version.

as a coherent calibration offset uncertainty, this error would not impact our estimate of the template error function. However, its estimate owes largely to the difficulty in identifying clean sequences with which to align the different fields, and it enters our cosmology pipeline as an uncertainty on the object flux. In this sense, we feel it is appropriate to account for this source of error in our assessment of the template error function. Doing so brings the $g$ and $u$ bands into much better agreement, though they remain $\sim 3$ per cent apart. As a large fraction of the variance in the $u$-band measurements in Fig. 17 appears to be from the photometric calibration, and not the 
template error, we drop the $u$ band while calibrating the template error function.

Our modified template error function is simply a wavelengthdependent scaled version of the one shipped with EAzY, fit to the $g$ and $r$-band data and using the following functional form to allow a smooth extrapolation through the rest-frame UV wavelength range,

$A_{\text {new }}=A *\left(1-\operatorname{erf}\left(\left(\lambda-\lambda_{0}\right) / 1000\right)\right)+1$,

where $A$ and $\lambda_{0}$ are free parameters to be fit and erf() is the Gauss error function. We use $A=0.8$ and $\lambda=3000 \AA$ to produce our modified template error function (dotted line in Fig. 18). Following the photometric redshift performance tests (Section 5.3), we compute the predicted template error function for a sample of high-redshift galaxies with 3D-HST grism redshifts, probed by the $g, r, i$, and $z$ bands over the wavelength range $2000<\lambda<7000 \AA$. We find that, although these data are noisier than those shown for PRIMUS, the average of the four bands agrees well with our modified template error function.

The final step is to find the correct scaling of the template error function. EAzY returns the best $\chi^{2}$ value at each redshift, meaning that we cannot use a formal marginalization over the SED component amplitudes to derive the redshift posterior. This choice improves EAzY's speed and scalability, without significant detriment to the single-value best redshift estimates (point estimates). However, the approximation of the multidimensional likelihood into one dimension in this way causes a flattening of the final redshift PDFs, resulting in overbroadening. A compromise can be found by reducing the overall amplitude of the template error function, while retaining its influence on the relative likelihood at different redshifts. In our photo-z run we use a 50 per cent amplitude.

\subsection{Photometric redshift performance}

Ideal photo-z will be both highly predictive, i.e. allow a point redshift estimate that is close to the true value, and have meaningful and accurate PDFs. The former has traditionally been the focus in assessing photo-z performance for deep extragalactic science (e.g. Dahlen et al. 2013), though the use of redshift PDFs in extragalactic science has become increasingly common (Wake et al. 2011; Hartley et al. 2013; Etherington et al. 2017) and with it a necessity to assess the accuracy of them. A number of metrics have been developed to measure and compare different photo-z predictions (see e.g. Zhan \& Knox 2006; Bordoloi, Lilly \& Amara 2010; Dahlen et al. 2013; Sánchez et al. 2014; Bonnett et al. 2016; Euclid Collaboration et al. 2020; Schmidt et al. 2020). Most require a sample of objects with high-confidence spectroscopic redshifts, though methods have also been developed to infer performance purely statistically (Quadri et al. 2008). The most suitable performance metrics to measure will clearly depend upon the science goals of interest. It is beyond the scope of this work to be exhaustive in our tests, but to aid comparison with other data sets we choose the most commonly used subset that nevertheless cover the broad classes of use cases. The remainder of this subsection is split into those that concern how predictive our point redshifts are, and those that estimate the accuracy of our PDFs.

\subsubsection{Point prediction performance}

We assess the quality of our best single-value redshift estimates through three metrics: mean bias $\left(\mathbb{E} \Delta_{z}\right.$, where $\Delta_{z}=\left(z_{\mathrm{ph}}-z_{\mathrm{sp}}\right) /(1$ $\left.+z_{\mathrm{sp}}\right)$ ), Normalized Absolute Median Deviation (NMAD),

$\mathrm{NMAD}=1.48 \times \operatorname{median}\left(\left|\Delta_{z}\right|\right)$ and outlier fraction. Outliers are defined as $\left|\Delta_{z}\right| \geq 0.12$. We also compute bias and NMAD with outliers excluded. Results are shown in Table 7 for five different samples:

(i) The high-confidence spectroscopic sample described in Section 5.1, excluding PRIMUS, 3D-HST and objects flagged as AGN.

(ii) This same spectroscopic sample, but with a set of weights applied based on $i$-band magnitude, such that their weighted magnitude histogram matches the Deep-Fields catalogue cut to $17<i<24$ (see Fig. 19).

(iii) A flux limited sample, $17<i^{\prime}<23$, with selection magnitude and reference redshift taken from Alarcon et al. (2020).

(iv) A flux limited sample, $17<i<24$, with reference redshift taken from Laigle et al. (2016).

(v) A flux limited sample, $17<i<26$, with reference redshift taken from Laigle et al. (2016).

The total number used, mean redshift and mean $i$-band magnitude are also reported and we show a comparison of the point redshift estimate against spectroscopic redshift in the upper panel of Fig. 20.

Performance is very good up to redshift, $z=1.5$, and down to 24th magnitude, with a very tight core of objects quantified by an overall NMAD of less than 3.5 per cent, and well-controlled mean bias. However, the fraction of outliers rises quite quickly between 23rd and 24th magnitude, and even more so at progressively fainter magnitudes. Beyond $z=1.5$, precise redshifts require an intermediate band between the $z$-band and $J$-band to constrain the $4000 \AA$ break wavelength (e.g. VISTA $Y$ band), which is not included in our catalogue. Performance is improved slightly beyond $z \sim 2$, when the break comfortably enters the $J$ band, but by these redshifts there are few objects with strong $4000 \AA$ break features. As a result the dispersion in $z_{\mathrm{ph}}-z_{\mathrm{sp}}$ becomes larger at $z>1.5$, until galaxies bright in the $i$-band become $u$-band drop-outs and an effective Lyman break selection becomes possible $(z>3)$. At these high redshifts we see the typical degeneracy caused by confusion between the Lyman break and $4000 \AA$ break in what are otherwise largely featureless SEDs. Although outliers in the sense of point redshift estimates, many of these objects will have redshift PDFs that capture the degeneracy.

It is interesting to compare the relative metric performances between the weighted spectroscopic sample and the L16 sample under the same magnitude cuts. The spectroscopic sample is highly heterogeneous, with selections targeting emission line galaxies, luminous red galaxies, high-redshift galaxies, and other particular galaxy subsamples. Moreover, despite the fact that we have removed AGNs where labelled, it is by no means certain that we have reduced their number to a fair sampling rate. In addition, a small fraction of the spectroscopic objects are likely to be blended sources, perhaps with redshift determined from emission lines that do not represent the bulk of the galaxy light (Masters et al. 2019), and may contain other subtle selection biases (Hartley et al. 2020). Conversely, a small fraction of the L16 redshifts are incorrect, even for these relatively bright sources (see Laigle et al. 2016, Section 4.3). Though the differences in the metric results are not large, they suggest that perhaps the spectroscopic sample is under-represented by a galaxy sub-population that have relatively weak predictions, but overrepresented by the type of objects that cause catastrophic failures in redshift determination. Meanwhile, the results of the sample matched to Alarcon et al. (2020) show that our point estimates are exceptional for an 8-band catalogue at $i<23$. 
Table 7. Photo- $z$ metrics: Mean bias $\left(\left(z_{\mathrm{ph}}-z_{\mathrm{sp}}\right) /\left(1+z_{\mathrm{sp}}\right)\right)$, Normalized Median Absolute Deviation, outlier rate and Kullback-Leibler divergence of the probability integral transform histogram with respect to an ideal flat distribution (see text for details).

\begin{tabular}{|c|c|c|c|c|c|}
\hline & Spectroscopic & $\begin{array}{l}\text { Weighted spectroscopic, } \\
\qquad 17<i<24\end{array}$ & $\begin{array}{c}\text { Alarcon20, } \\
17<i^{\prime}<23\end{array}$ & $\begin{array}{l}\text { Laigle16, } \\
17<i<24\end{array}$ & $\begin{array}{l}\text { Laigle16, } \\
17<i<26\end{array}$ \\
\hline Overall NMAD & 0.0223 & 0.0301 & 0.0228 & 0.0339 & 0.0736 \\
\hline NMAD, no outliers & 0.0205 & 0.0264 & 0.0219 & 0.0302 & 0.0467 \\
\hline Outlier rate - point & 6.3 per cent & 9.5 per cent & 2.9 per cent & 8.4 per cent & 26.1 percent \\
\hline Mean bias, $\mathbb{E} \Delta z$ & 0.0147 & 0.0097 & 0.0136 & 0.0131 & 0.0551 \\
\hline Mean bias, no outliers & 0.0027 & 0.0057 & 0.0019 & 0.0094 & 0.0096 \\
\hline $\begin{array}{l}\text { PIT KL-divergence } \\
\text { Outlier rate - PDF }\end{array}$ & $\begin{array}{l}1.2 \times 10^{-4} \text { Hart } \\
5.0 \text { percent }\end{array}$ & $\begin{array}{l}3.2 \times 10^{-4} \text { Hart } \\
6.2 \text { percent }\end{array}$ & $\begin{array}{c}1.2 \times 10^{-4} \text { Hart } \\
3.3 \text { per cent }\end{array}$ & $\begin{array}{l}5.0 \times 10^{-4} \text { Hart } \\
5.8 \text { per cent }\end{array}$ & $\begin{array}{l}9.3 \times 10^{-4} \text { Hart } \\
6.2 \text { percent }\end{array}$ \\
\hline $\mathrm{N}$ objects & 34948 & 32956 & 30737 & 72391 & 222231 \\
\hline Mean redshift & 0.693 & 0.898 & 0.642 & 0.846 & 1.294 \\
\hline Mean $i$-band mag. & 21.49 & 22.72 & 21.87 & 22.75 & 24.33 \\
\hline
\end{tabular}

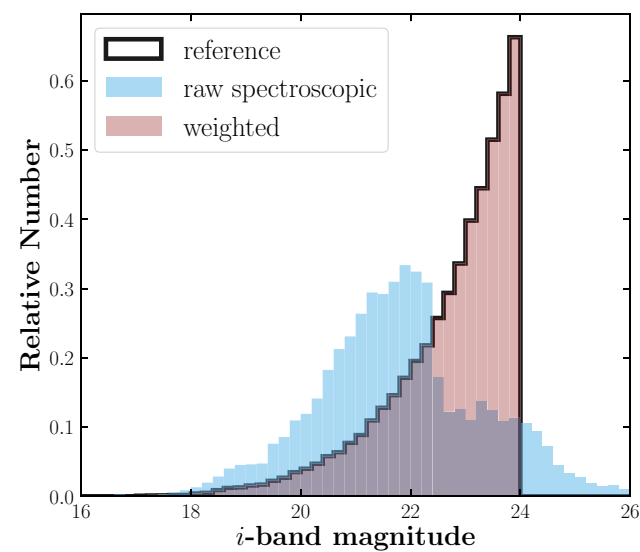

Figure 19. $i$-band magnitude distribution for objects in our collated spectroscopic redshift sample, together with the flux-limited Deep-Fields sample at $i$ $<24$ and weighted spectroscopic distribution which matches the flux-limited sample, by construction.

\subsubsection{PDF performance}

To assess the accuracy of the EAzY PDFs we turn to the Probability Integral Transform (PIT) distribution (Dawid 1984). PIT is defined as the value of the cumulative distribution function evaluated at the true (spectroscopic/many-band photo- $z$ ) redshift,

$P I T=\int_{-\infty}^{z \mathrm{sp}} p(z) \mathrm{d} z$

and its distribution over a sample is being increasingly used as a key test of the accuracy of redshift PDF calibration. It can also be used as part of an additional post-processed calibration if the PDFs are found to be inaccurate (Bordoloi et al. 2010; Hoyle et al. 2018), but we do not use it for this purpose. If a set of 1D PDFs are well calibrated then their distribution of PIT values should be indistinguishable from a set of random draws from a uniform distribution, $U(0,1)$. We show the PIT distribution for the (unweighted) spectroscopic sample in the middle panel of Fig. 20. For some objects the spectroscopic redshift lies entirely outside of the photo-z PDF, either at higher or lower redshift. These objects we define as outliers and contribute to the extreme ends of the histogram, but we exclude them when quantifying the shape of the PIT distribution. Our metrics in testing our photo-z PDFs are the outlier fraction, and the Kullback-Leibler (KL) divergence of the PIT histogram, relative to perfect performance
- i.e. relative to a flat distribution:

$\mathrm{KL}=\sum p \log _{10}(p / q)$,

where $p$ is the histogram of PIT values, and $q$ a flat distribution. The KL divergence is a measure of information which, in the case of log base-10, carries the unit, Hart. As a final, visual, test of our PDFs, we show the redshift distribution estimated via summing the individual redshift PDFs and comparing it with a kernel density estimate (KDE) of their spectroscopic redshifts. The bandwidth of the KDE was chosen such that the smoothing kernel reflects the value of NMAD. While a sum of PDFs is by no means the best estimator of the redshift distribution (for a discussion, see Malz \& Hogg 2020), it is nevertheless commonly used in scientific analyses and thus warrants inclusion here.

The results of the two PDF metrics are shown in Table 7. Two trends stand out: the PDF outlier fraction is fairly insensitive to the sample used and does not climb at fainter magnitudes; the PIT KLdivergence increases as the sample magnitude limit is made fainter. The first observation tells us that the large outlier fraction found in the point redshift metrics at faint magnitudes (26.1 per cent for the deepest sample) is not due to catastrophic outliers of galaxies without appropriate models in the photo- $z$ set up, but rather represents a simple decrease in predictive power caused by a lack of information. In other words, the high point-redshift outlier fraction in these deeper samples does not suggest a fault in redshift determination, but a limit to the precision that can be achieved at faint magnitudes with our 8-band data.

The rise in KL-divergence for faint samples represents a possible weakness in the accounting of the error budget of these objects. Indeed, the PIT histogram for our faintest sample (not shown) has a clear but shallow concave shape, which is typically due to slightly underdispersed PDFs (i.e. underestimated uncertainties), and evidence of a small bias in the sense that the PDFs are shifted towards higher redshift. The PIT histogram for our combined spectroscopic sample shows the opposite behaviour: the slight deficit of objects with PIT $\sim 0.1-0.3$ indicates a very small bias in the sense that the PDFs are systematically too low in redshift. Note that this possible PDF bias for the spectroscopic sample is in the opposite sense of the point redshift bias. The solution to this minor calibration defect at faint magnitudes is unclear, and it is likely that we would not be able to incorporate it within the existing EAzY software. Possible solutions may include fully incorporating the band-to-band flux covariance, computing a magnitude dependent template error function or applying ad hoc contributions to the flux errors as a function of magnitude. 

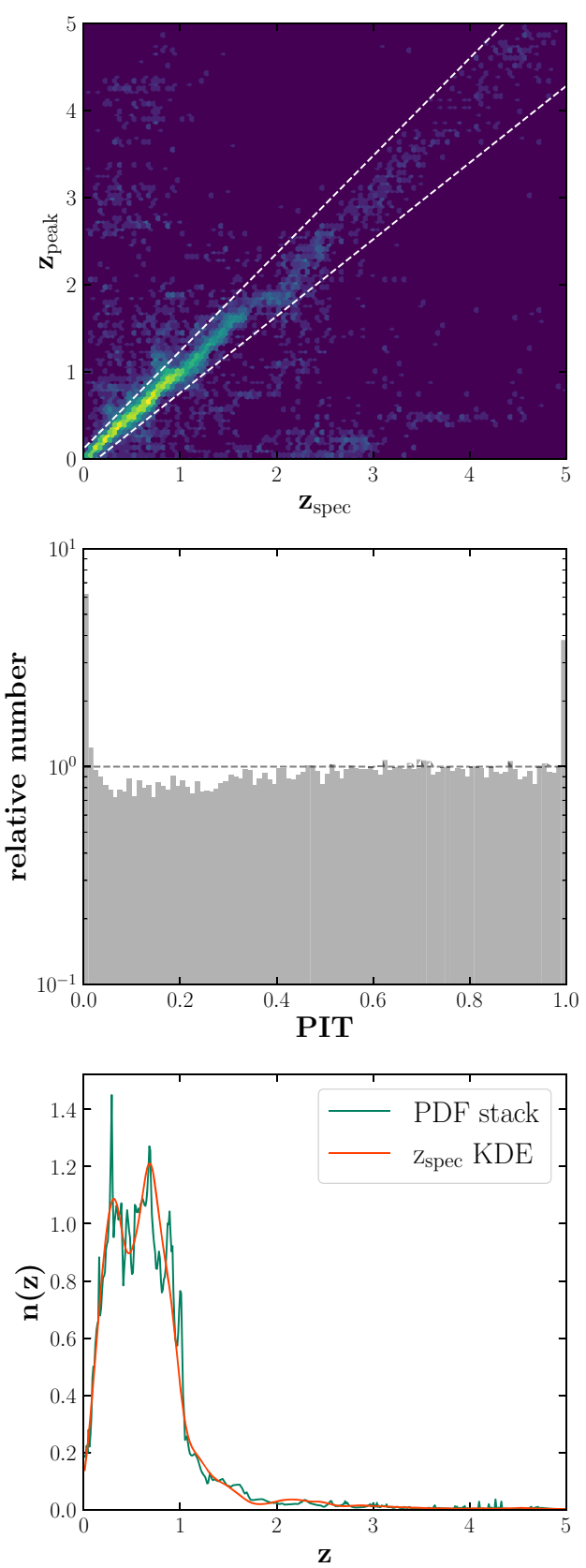

Figure 20. Photometric redshift performance for the (unweighted) spectroscopic redshift sample. Top: comparison of spectroscopic and best photometric redshift estimate. The colour scale corresponds to the log density of points, i.e. the vast majority of objects lie at $z<1$ and have very accurate photo- $z$ estimates. Middle: Probability Integral Transform histogram. A flat histogram represents a probabilistically calibrated data set. Deviations from flatness indicate over or underdispersed PDFs, while a slope may indicate an overall bias. Peaks at either end of the histogram quantify the fraction of outliers, where the spectroscopic redshift lies entirely outside of the photo- $z$ PDF. Our results compare very favourably to similar plots in the literature (Euclid Collaboration 2020; Schmidt et al. 2020). Bottom: n(z) recovery via coaddition of photo- $z$ PDFs, compared with a Kernel Density Estimate of their spectroscopic redshifts.

\section{CONCLUSIONS}

In this paper, we have described the construction of the DES Deep-Fields images and the catalogue drawn from a subset of those images for the survey's main three-year cosmology analysis (covering four fields of $\sim 1.5 \mathrm{sq}$. deg. apiece). Through combining observations from our SN survey, community data and additional dedicated observations of the COSMOS field and SN fields, we have constructed images that comprise ten times the exposure time of the main survey, and that have seeing FWHM better than 50 per cent or more of the WS data set. We have combined these images with nearIR data from two VISTA programmes: VIDEO and UltraVISTA, to produce a data set spanning the $u$ to $K s$ wavelength range. The final catalogue is based on detection from the average of the $r, i$ and $z$ bands, numbering 2.8 million sources, which is reduced to 1.7 million after extensive and careful masking is applied. Deblended source photometry and forced photometry measurements of individual bands were performed with the fitvd model-fitting code, which ensures consistent colours for our extracted sources.

We have presented tests of the source extraction, finding $>$ 90 per cent completeness to $i=25$ th magnitude in each field and agreement in number counts at the level of 90 per cent or better across the magnitude range of relevance for DES Y3 cosmology. Our PSF modelling accuracy has been demonstrated to be within 1 per cent error across the required range for PSF construction in all bands, which is crucial for measuring accurate source colours. We have further performed a fine tuning of the relative colours and photometric zero-points between our four fields, and anchored the calibration to our main survey data. Our final estimated zero-point uncertainties are found to be 0.5 per cent in the key DES bands $(g$, $r, i$, and $z), 0.8$ per cent in the VISTA bands $(J, H$, and $K s)$, and 5.5 per cent in the $u$ band.

With our calibrated catalogue we have shown the performance of a colour-based star-galaxy separation method, using morphologydetermined stars in the HST COSMOS data as ground truth. We have found that we are able to efficiently separate the two object classes to magnitudes as faint as $i \sim 22$, with stellar completeness degrading to 90 percent by $i=22.5$. Finally, we have detailed the production of photometric redshifts, using the EAzY code, including additional zero-point corrections that are specific to the template set employed, and a small calibration adjustment to the template error function used. The resulting photo-z show excellent performance with respect to spectroscopic redshifts and highly accurate photo$\mathrm{z}$ from COSMOS (Laigle et al. 2016) and PAUS (Alarcon et al. 2020), to magnitudes as faint as $i=24$. At fainter magnitudes, the performance understandably declines, but remains useful.

The DES Deep-Fields catalogue is suitable for use in a plethora of stand-alone scientific analyses, from exploration of the evolution of the stellar mass function (Gschwend et al., in preparation) to studies of the host galaxies of transient events (Meldorf et al., in preparation) and multidimensional derivation of galaxy property posteriors via machine learning (Mucesh et al. 2021). However, its primary motivation is for use in the DES cosmology analysis, combining weak lensing shear, galaxy clustering and galaxy-galaxy lensing (DES collaboration et al. 2021). The principal roles of the Deep-Fields data for the cosmology analysis are: (1) measuring the transfer function of the survey - i.e. the characteristics of the sources we extract from our main DES survey data relative to the input truth (for the Y3 implementation, see Everett et al. 2020); (2) building a high-dimensional self-organizing map (SOM), in which the redshift distributions of each cell are intrinsically narrow with respect to the SOM built from the 4-band data of the main survey 
(for the Y3 implementation, see Myles et al. 2020). The Y3 image simulations used to calibrate the weak lensing shear also utilises a variant of the Deep-Fields catalogue based on detections and fits to morphological parameters on HST imaging in COSMOS and fluxes estimated on the DECam griz imaging (MacCrann et al. 2020). Other advantages in building these data include being able to use a datadriven prior on the moments of galaxy light distributions for galaxy shear measurement (Bernstein \& Armstrong 2014a; Bernstein et al. 2016), possible because of our selection of input images with good seeing ( $<1.0$ arcsec in riz bands).

The coming few years will see the second full public release of DES data (DR2), including data from all six years of DES observations. Shortly thereafter will follow our cosmology analyses with these data, and as part of that we will need to build a DeepFields data set that is able to support the increased statistical precision that the final survey data will provide. With respect to the catalogue presented in this work, that data set will need to be incrementally deeper, but crucially also cover a wider area. Taking advantage of our other SN fields and the recent VISTA Extragalactic Infrared Legacy Survey ${ }^{17}$ (VEILS; Banerji et al., in preparation), we will be able to double our area, thereby reducing sample variance uncertainties and adding additional sources of spectroscopy. Finally, in the spirit of establishing a legacy value for the DES Deep Fields, we will produce a catalogue based on the DEEPEST level of SN coadd images, across all ten $\mathrm{SN}$ pointings.

Software: SExtractor (Bertin \& Arnouts 1996), PSFEx (Bertin 2011), SCAMP (Bertin 2006), SWarp (Bertin et al. 2002; Bertin 2010).

\section{ACKNOWLEDGEMENTS}

We thank our referee, Adriano Fontana, for their careful reading of our manuscript and suggestions that improved the clarity of our text.

AC acknowledges support from NASA grant 15-WFIRST150008. IH, acknowledges support from the European Research Council in the form of a Consolidator Grant with number 681431 and from the Beecroft Trust.

Funding for the DES Projects has been provided by the U.S. Department of Energy, the U.S. National Science Foundation, the Ministry of Science and Education of Spain, the Science and Technology Facilities Council of the United Kingdom, the Higher Education Funding Council for England, the National Center for Supercomputing Applications at the University of Illinois at Urbana-Champaign, the Kavli Institute of Cosmological Physics at the University of Chicago, the Center for Cosmology and Astro-Particle Physics at the Ohio State University, the Mitchell Institute for Fundamental Physics and Astronomy at Texas A\&M University, Financiadora de Estudos e Projetos, Fundação Carlos Chagas Filho de Amparo à Pesquisa do Estado do Rio de Janeiro, Conselho Nacional de Desenvolvimento Científico e Tecnológico and the Ministério da Ciência, Tecnologia e Inovação, the Deutsche Forschungsgemeinschaft and the Collaborating Institutions in the Dark Energy Survey.

The Collaborating Institutions are Argonne National Laboratory, the University of California at Santa Cruz, the University of Cambridge, Centro de Investigaciones Energéticas, Medioambientales y Tecnológicas-Madrid, the University of Chicago, University College London, the DES-Brazil Consortium, the University of Edinburgh, the Eidgenössische Technische Hochschule (ETH) Zürich, Fermi

\footnotetext{
${ }^{17}$ https://people.ast.cam.ac.uk/mbanerji/VEILS
}

National Accelerator Laboratory, the University of Illinois at UrbanaChampaign, the Institut de Ciències de l'Espai (IEEC/CSIC), the Institut de Física d'Altes Energies, Lawrence Berkeley National Laboratory, the Ludwig-Maximilians Universität München and the associated Excellence Cluster Universe, the University of Michigan, NFS's NOIRLab, the University of Nottingham, The Ohio State University, the University of Pennsylvania, the University of Portsmouth, SLAC National Accelerator Laboratory, Stanford University, the University of Sussex, Texas A\&M University, and the OzDES Membership Consortium.

Based in part on observations at Cerro Tololo Inter-American Observatory at NSF's NOIRLab (NOIRLab Prop. ID 2012B-0001; PI: J. Frieman), which is managed by the Association of Universities for Research in Astronomy (AURA) under a cooperative agreement with the National Science Foundation.

The DES data management system is supported by the National Science Foundation under Grant Numbers AST-1138766 and AST-1536171. The DES participants from Spanish institutions are partially supported by MICINN under grants ESP2017-89838, PGC2018-094773, PGC2018-102021, SEV-2016-0588, SEV-20160597, and MDM-2015-0509, some of which include ERDF funds from the European Union. IFAE is partially funded by the CERCA program of the Generalitat de Catalunya. Research leading to these results has received funding from the European Research Council under the European Union's Seventh Framework Program (FP7/2007-2013) including ERC grant agreements 240672, 291329, and 306478. We acknowledge support from the Brazilian Instituto Nacional de Ciência e Tecnologia (INCT) do e-Universo (CNPq grant 465376/2014-2).

This manuscript has been authored by Fermi Research Alliance, LLC under Contract No. DE-AC02-07CH11359 with the $\langle 0$ :fund ing-source 3:href="http://dx.doi.org/10.13039/100000015" $\rangle$ U.S. De partment of Energy $\langle/ 0$ :funding-source $\rangle$, OfficeofScience, OfficeofH ighEnergyPhysics.

\section{DATA AVAILABILITY}

The data underlying this article and that were used to build the processed data products were accessed from NOAO (https://www. noao.edu/). The derived data will be released publicly through the DES collaboration at https://des.ncsa.illinois.edu/releases/ upon the completion of the cosmology analysis for which they are used.

\section{REFERENCES}

Abbott T. M. C. et al., 2018, ApJS, 239, 18

Abolfathi B. et al., 2018, ApJS, 235, 42

Aihara H. et al., 2018, PASJ, 70, S4

Aihara H. et al., 2019, PASJ, 71, 114

Alarcon A. et al., 2020, MNRAS, 501, 6103

Baldry I. K. et al., 2018, MNRAS, 474, 3875

Bazin G. et al., 2011, A\&A, 534, A43

Benítez N., 2000, ApJ, 536, 571

Bernstein G. M., Armstrong R., 2014a, MNRAS, 438, 1880

Bernstein G. M., Armstrong R., 2014b, MNRAS, 438, 1880

Bernstein G. M., Armstrong R., Krawiec C., March M. C., 2016, MNRAS, 459, 4467

Bertin E., 2006, in Gabriel C., Arviset C., Ponz D., Enrique S., eds, ASP Conf. Ser. Vol. 351, Astronomical Data Analysis Software and Systems XV. Astron. Soc. Pac., San Francisco, p. 112

Bertin E., 2010, Astrophysics Source Code Library. record ascl:1010.068 
Bertin E., 2011, in Evans I. N., Accomazzi A., Mink D. J., Rots A. H., eds, ASP Conf. Ser. Vol. 442, Astronomical Data Analysis Software and Systems XX. Astron. Soc. Pac., San Francisco, p. 435

Bertin E., Arnouts S., 1996, A\&AS, 117, 393

Bertin E., Mellier Y., Radovich M., Missonnier G., Didelon P., Morin B., 2002, in Bohlender D. A., Durand D., Handley T. H., eds, ASP Conf. Ser. Vol. 281, Astronomical Data Analysis Software and Systems XI. Astron. Soc. Pac., San Francisco, 228

Bonnett C. et al., 2016, Phys. Rev. D, 94, 042005

Bordoloi R., Lilly S. J., Amara A., 2010, MNRAS, 406, 881

Bouwens R. J., Illingworth G. D., Franx M., Ford H., 2007, ApJ, 670, 928

Bowler R. A. A., Jarvis M. J., Dunlop J. S., McLure R. J., McLeod D. J., Adams N. J., Milvang-Jensen B., McCracken H. J., 2020, MNRAS, 493, 2059

Bradshaw E. J. et al., 2013, MNRAS, 433, 194

Brammer G. B., van Dokkum P. G., Coppi P., 2008, ApJ, 686, 1503

Brown M. J. I. et al., 2014, ApJS, 212, 18

Buchs R. et al., 2019, MNRAS, 489, 820

Burke D. L. et al., 2018, AJ, 155, 41

Chambers K. C. et al., 2016, preprint(arXiv:1612.05560)

Cohen J. G., 2002, ApJ, 567, 672

Coil A. L. et al., 2004, ApJ, 609, 525

Coil A. L. et al., 2011, ApJ, 741, 8

Colless M. et al., 2001, MNRAS, 328, 1039

Conroy C., Gunn J. E., 2010, FSPS: Flexible Stellar Population Synthesis

Cool R. J. et al., 2013, ApJ, 767, 118

Cooper M. C. et al., 2012, MNRAS, 425, 2116

Coppin K. et al., 2006, MNRAS, 372, 1621

Czekaj M. A., Robin A. C., Figueras F., Luri X., Haywood M., 2014, A\&A, 564, A102

D'Andrea C. B. et al., 2018, preprint(arXiv:1811.09565)

Daddi E., Cimatti A., Renzini A., Fontana A., Mignoli M., Pozzetti L., Tozzi P., Zamorani G., 2004, ApJ, 617, 746

Dahlen T. et al., 2013, ApJ, 775, 93

Dalton G. B. et al., 2006, in McLean; I. S., Masanori I., eds, Proc. SPIE Conf. Ser. Vol. 6269, Ground-based and Airborne Instrumentation for Astronomy. SPIE, Bellingham, p. 62690X

Davidzon I. et al., 2017, A\&A, 605, A70

Dawid A. P., 1984, J. R. Stat. Soc. Ser. A (General), 147, 278

DES Collaboration, 2021, Dark Energy Survey Year 3 Results: Cosmological Constraints from Galaxy Clustering and Weak Lensing. preprint (arXiv:2105.13549)

DES Collaboration, 2016, MNRAS, 460, 1270

Drlica-Wagner A. et al., 2018, ApJS, 235, 33

Elmegreen D. M., Elmegreen B. G., Ravindranath S., Coe D. A., 2007, ApJ, 658,763

Emerson J. P., Sutherland W. J., McPherson A. M., Craig S. C., Dalton G. B., Ward A. K., 2004, The Messenger, 117, 27

Etherington J. et al., 2017, MNRAS, 466, 228

Euclid Collaboration, 2020, A\&A, 644, A31

Everett S. et al., 2020, preprint(arXiv:2012.12825)

Fitzpatrick E. L., 1999, PASP, 111, 63

Flaugher B. et al., 2015, AJ, 150, 150

Flaugher B., 2005, Int. J. Mod. Phys. A, 20, 3121

Foucaud S. et al., 2007, MNRAS, 376, L20

Furusawa H. et al., 2008, ApJS, 176, 1

Gaia Collaboration, 2016, A\&A, 595, A1

Galametz A. et al., 2013, ApJS, 206, 10

Galametz A. et al., 2018, MNRAS, 475, 4148

Garilli B. et al., 2014, A\&A, 562, A23

Giavalisco M. et al., 2004, ApJ, 600, L93

González-Fernández C. et al., 2018, MNRAS, 474, 5459

Guo Y. et al., 2013, ApJS, 207, 24

Hartley W. G. et al., 2013, MNRAS, 431, 3045

Hartley W. G. et al., 2020, MNRAS, 496, 4769

Hartley W. G., Conselice C. J., Mortlock A., Foucaud S., Simpson C., 2015, MNRAS, 451, 1613

Hasinger G. et al., 2018, ApJ, 858, 77
Herenz E. C. et al., 2017, A\&A, 606, A12

Hoyle B. et al., 2018, MNRAS, 478, 592

Ilbert O. et al., 2013, A\&A, 556, A55

Jarvis M. et al., 2016, MNRAS, 460, 2245

Jarvis M. J. et al., 2013, MNRAS, 428, 1281

Jones D. H. et al., 2009, MNRAS, 399, 683

Joye W. A., Mandel E., 2003, in Payne H. E., Jedrzejewski R. I., Hook R. N., eds, New Features of SAOImage DS9. p. 489

Kashikawa N. et al., 2004, PASJ, 56, 1011

Kim J.-W., Edge A. C., Wake D. A., Stott J. P., 2011, MNRAS, 410, 241

Koekemoer A. M. et al., 2011, ApJS, 197, 36

Kohonen T., 1982, Biol. Cybern., 43, 59

Kovač K. et al., 2010, ApJ, 718, 86

Kriek M. et al., 2015, ApJS, 218, 15

Kron R. G., 1980, ApJS, 43, 305

Kuijken K. et al., 2019, A\&A, 625, A2

Lahav O., Calder L., Mayers J., Freiman J., 2020, The Dark Energy Survey: The Story of a Cosmological Experiment. World Scientific, Singapore

Laigle C. et al., 2016, ApJS, 224, 24

Lane K. P. et al., 2007, MNRAS, 379, L25

Lang D., Hogg D. W., Mykytyn D., 2016, Astrophysics Source Code Library. record ascl: 1604.008

Lawrence A. et al., 2007, MNRAS, 379, 1599

Le Fèvre O. et al., 2005, A\&A, 439, 845

Le Fèvre O. et al., 2013, A\&A, 559, A14

Le Fèvre O. et al., 2015, A\&A, 576, A79

Leauthaud A. et al., 2007, ApJS, 172, 219

Lee B. et al., 2013, ApJ, 774, 47

Lidman C. et al., 2020, MNRAS, 496, 19

Lilly S. J. et al., 2007, ApJS, 172, 70

Lilly S. J. et al., 2009, ApJS, 184, 218

Lonsdale C. J. et al., 2003, PASP, 115, 897

Lotz J. M., Madau P., Giavalisco M., Primack J., Ferguson H. C., 2006, ApJ, 636, 592

MacCrann N., Blazek J., Jain B., Krause E., 2020, MNRAS, 491, 5498

Maltby D. T. et al., 2019, MNRAS, 489, 1139

Malz A. I., Hogg D. W., 2020, preprint(arXiv:2007.12178)

Masters D. C. et al., 2019, ApJ, 877, 81

Masters D. C., Stern D. K., Cohen J. G., Capak P. L., Rhodes J. D., Castander F. J., Paltani S., 2017, ApJ, 841, 111

Masters D. et al., 2015, ApJ, 813, 53

McCracken H. J. et al., 2012, A\&A, 544, A156

McLure R. J. et al., 2013, MNRAS, 428, 1088

McLure R. J. et al., 2018, MNRAS, 479, 25

Merlin E. et al., 2015, A\&A, 582, A15

Merlin E. et al., 2016, A\&A, 595, A97

Momcheva I. G. et al., 2016, ApJS, 225, 27

Momose R. et al., 2020, ApJ, 909, 117

Morganson E. et al., 2018, PASP, 130, 074501

Mortlock A. et al., 2015, MNRAS, 447, 2

Mucesh S., 2021, A machine learning approach to galaxy properties: joint redshift-stellar mass probability distributions with Random Forest, MNRAS, 502, 2770

Muzzin A. et al., 2012, ApJ, 746, 188

Muzzin A. et al., 2013, ApJS, 206, 8

Myles J. et al., 2020, MNRAS, 505, 4249

Neilsen E. H. Jr, Annis J. T., Diehl H. T., Swanson M. E., D'Andrea C., Kent S., Drlica-Wagner A., 2019, doi:10.2172/1574836

Neilsen E. H. Jr, Bernstein G., Gruendl R., Kent S., 2016, doi:10.2172/1250877

Quadri R. F., Williams R. J., Lee K.-S., Franx M., van Dokkum P., Brammer G. B., 2008, ApJ, 685, L1

Rasmussen C. E., Williams C. K. I., 2005, Gaussian Processes for Machine Learning (Adaptive Computation and Machine Learning). The MIT Press, USA

Rest A. et al., 2014, ApJ, 795, 44

Robin A., Creze M., 1986, A\&A, 157, 71

Rowe B. T. P. et al., 2015, Astron. Comput., 10, 121 
Rudnick G. et al., 2003, ApJ, 599, 847

Sánchez C. et al., 2014, MNRAS, 445, 1482

Sánchez C., Bernstein G. M., 2019, MNRAS, 483, 2801

Schlafly E. F., Finkbeiner D. P., 2011, ApJ, 737, 103

Schlegel D. J., Finkbeiner D. P., Davis M., 1998, ApJ, 500, 525

Schmidt S. J. et al., 2020, MNRAS, 499, 1587

Scoville N. et al., 2007, ApJS, 172, 1

Seitz C., Schneider P., 1997, A\&A, 318, 687

Sevilla-Noarbe I., 2021, Dark Energy Survey Year 3 Results: Photometric Data Set for Cosmology. ApJS, 254, 24

Sherman S. et al., 2020, MNRAS, 491, 3318

Silverman J. D. et al., 2015, ApJS, 220, 12

Skrutskie M. F. et al., 2006a, AJ, 131, 1163

Skrutskie M. F. et al., 2006b, AJ, 131, 1163

Somerville R. S., Lee K., Ferguson H. C., Gardner J. P., Moustakas L. A., Giavalisco M., 2004, ApJ, 600, L171

Straatman C. M. S. et al., 2018, ApJS, 239, 27

Suchyta E. et al., 2016, MNRAS, 457, 786

Tanaka M. et al., 2018, PASJ, 70, S9

Tasca L. A. M. et al., 2017, A\&A, 600, A110

Wake D. A. et al., 2011, ApJ, 728, 46

Williams R. E. et al., 1996, AJ, 112, 1335

Williams R. J., Quadri R. F., Franx M., van Dokkum P., Labbé I., 2009, ApJ, 691,1879

Wisnioski E. et al., 2019, ApJ, 886, 124

Wittman D. M. et al., 2002, in Tyson J. A., Wolff S., eds, Proc. SPIE Conf. Ser. Vol. 4836, Survey and Other Telescope Technologies and Discoveries. SPIE, Bellingham, p. 73

Wright A. H. et al., 2019, A\&A, 632, A34

Zhan H., Knox L., 2006, ApJ, 644, 663

Zuntz J. et al., 2018, MNRAS, 481, 1149

\section{APPENDIX A: ASTROMETRY TEST WITH GAUSSIAN PROCESS}

At the start of this project, UltraVISTA data from the DR3 release ${ }^{18}$ was used. When comparing matched source positions between the UltraVISTA DR3 data set and the DECam images of the COSMOS field, significant offsets were found, as can be seen in the upper panels of Fig. A1. In order to place both data-sets into a common astrometric reference frame, we correct the offsets using a Gaussian Process (GP) model (a supervised machine learning method, see

\footnotetext{
${ }^{18}$ http://www.eso.org/sci/observing/phase3/data_releases/uvista_dr3.pdf
}

e.g. Rasmussen \& Williams 2005). We select bright stars from the matched UltraVISTA-DECam catalogue with the condition:

spread_model $+\frac{5}{3}$ spreaderr_model $<0.002$,

where spread_model and spreaderr_model are the corresponding columns from the DECam SEXTRACTOR catalogue. We then take eighty per cent of these sources and train a 2D Gaussian process for each RA offset and Declination offset between the sources' positions in the DECam and UltraVISTA images. The result of applying the correction from this Gaussian Process model for the 2D surfaces in RA and Dec. offset are shown in the left-hand column of Fig. A1. The centre column of Fig. A1 also shows the offsets for the remaining 20 per cent of the star sources which were reserved as a test set (i.e. which were not used in training the GP). As can be seen from the residual offsets, after correction with the GP, (centre row), the correction has significantly reduced the systematic offsets between the UltraVISTA and DECam positions. This can also be seen in the galaxy sample, in the right most column of Fig. A1. The GP used was implemented using GPy, ${ }^{19}$ with a Matern 5/2 kernel. Separate GPs were trained in the stripes in RA defined by the UltraVISTA deep and ultradeep regions. We implemented a number of improvements on this baseline configuration, including adding a white noise kernel to allow for stellar motions; including bright, compact galaxies as extra training points; and a number of different appropriate kernels. None of these led to significant improvements in the residual offsets after correction. In particular, tuning of kernels to allow for smaller scale variations in the offset correction surface led to overtraining, with smaller residuals for training sources, but larger residuals for the test sources. A summary of the improvements in the astrometry can be seen in the lower left-hand panel of Fig. A1.

Upon the release of UltraVISTA DR $4,{ }^{20}$ the initial offsets between UltraVISTA and DECam data were reduced to the same level as the post-correction residuals for DR3. We therefore proceed with the DR4 data without GP correction.

\footnotetext{
${ }^{19}$ https://github.com/SheffieldML/GPy

${ }^{20} \mathrm{http} / / /$ ultravista.org/release4/dr4_release.pdf
} 

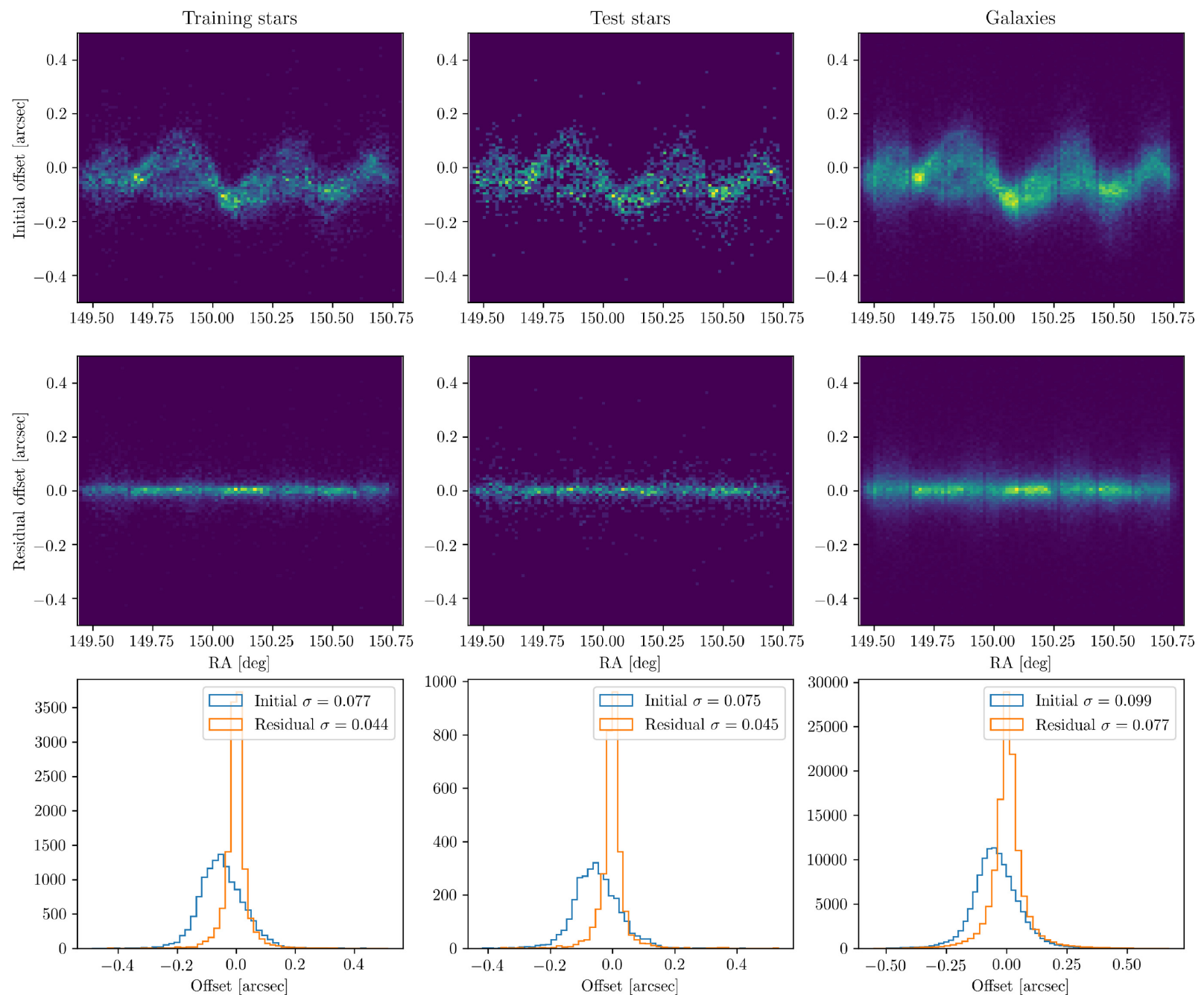

Figure A1. Residuals between UltraVISTA DR3 positions and DECam positions in the COSMOS field. Top row shows pre-correction offsets projected in the RA direction, middle row shows residuals following correction with the Gaussian Process model, lower row shows 1D histograms of the offsets both pre- and post-correction. Left column shows offsets for the sample of stars used to train the Gaussian Process, centre column shows offsets for the reserved test stars, and right column for the galaxy sources. 


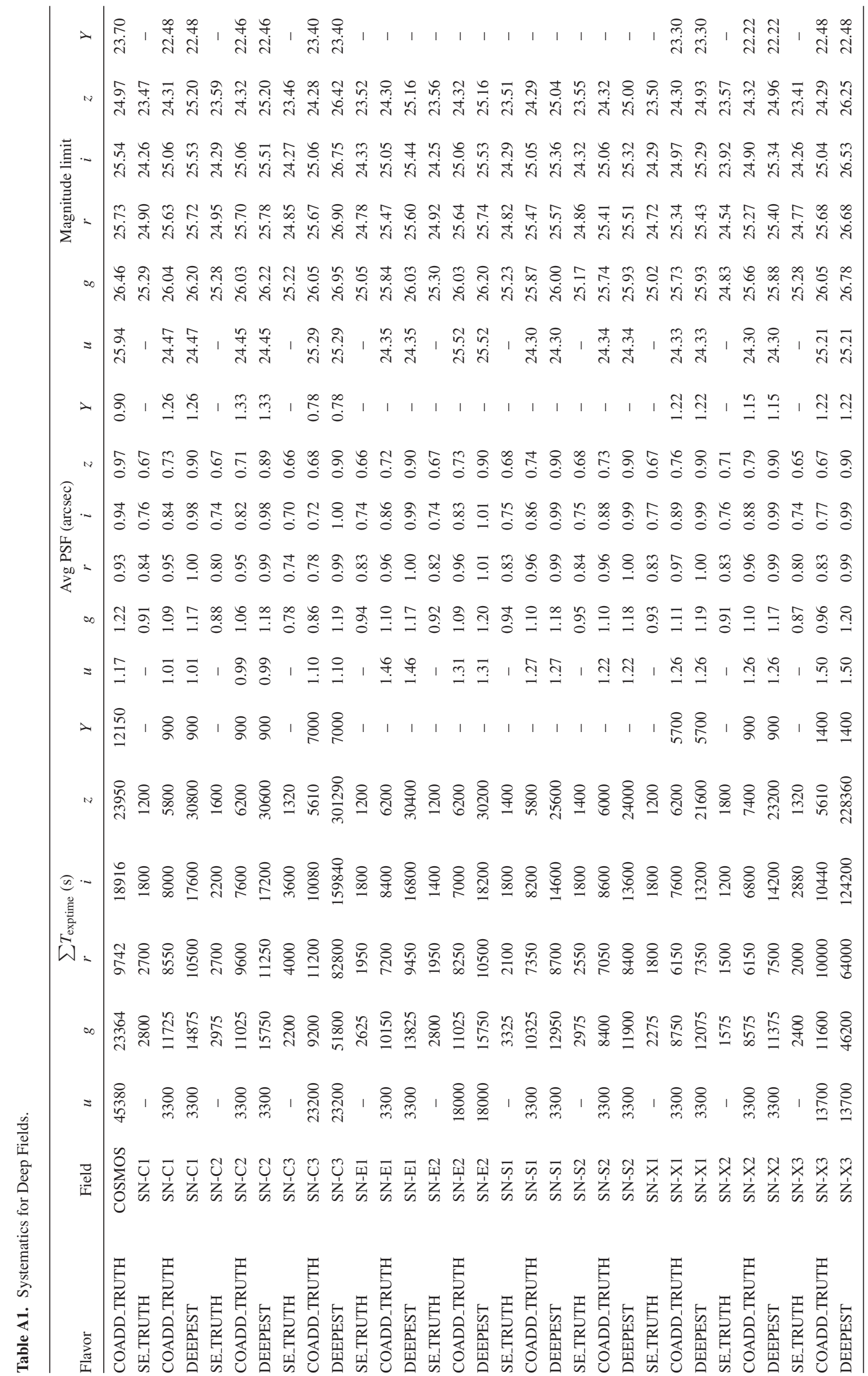




\section{APPENDIX B : ADDITIONAL DATA}

We expand on the description of the data in Section 2.1 by summarizing the quantities related to depth and image quality for the full set of 10 deep fields in Table A1.

In Fig. B1, we visualize the magnitude limits for the DECam data over each of the four fields chosen for the cosmology catalogue and for each of the filters ugrizY.

In Fig. B2, we plot the difference between the Deep-Fields catalogue PSF magnitudes in griz and the same objects found in HSC for $\mathrm{SN}-\mathrm{X} 3$ and COSMOS. In order to make the conversion between the DECam filters and the HSC filters, we used the Laigle et al. (2016) catalogue and Brown et al. (2014) templates to perform a guided interpolation from Subaru broad-bands to each of DES and HSC (see the appendix of Rudnick et al. 2003 for a description of the method used). With the magnitude differences and DECam colours per object, we fit a first-order polynomial to convert DECam bands to their HSC counterparts. The $r$-band conversion was poorly represented by a simple polynomial, with considerable dispersion at fixed colour.

For stellar sources, the $g, r$, and $z$ bands match within the expected uncertainties ( 0.5 per cent for the Deep-Fields catalogue and 1 per cent for HSC). The For the $i$ band, the COSMOS field show a 4 per cent difference between the two catalogues, the origin of which is unclear. There is a known issue in the HSC Cosmos photometry (Aihara et al. 2018), though this is not expected to result in an offset larger than 1.5 per cent. We use PSF magnitudes for this comparison and so do not expect the magnitudes of galaxies to match.
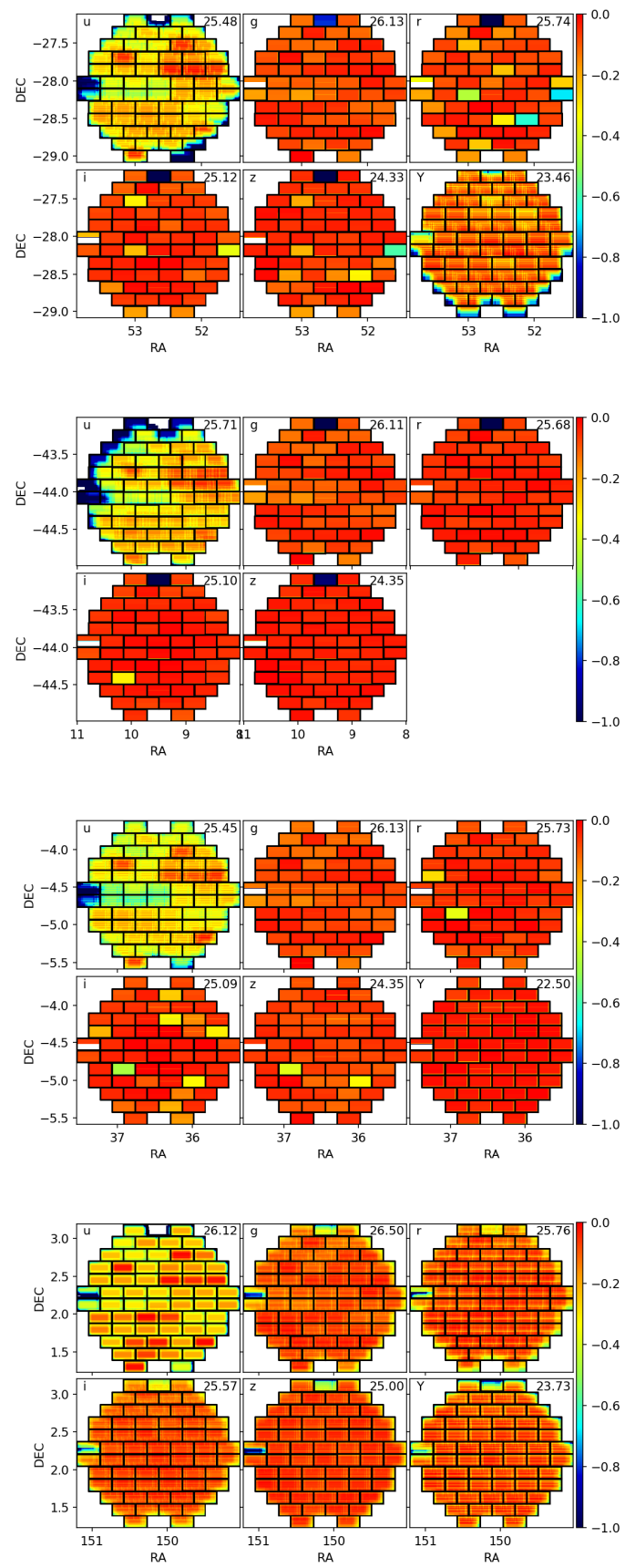

Figure B1. Magnitude limits calculated across each field. The fields (top to bottom) are $\mathrm{SN}-\mathrm{C} 3, \mathrm{SN}-\mathrm{E} 2, \mathrm{SN}-\mathrm{X} 3$, and COSMOS, respectively. The range shown for each panel is set to show variations (within $1 \mathrm{mag}$ ) of the maximum depth for a given field/band combination (the value for the maximum depth is given in the upper right corner of each panel). 



Figure B2. Comparison of PSF magnitudes between DES Deep Fields and HSC. DES magnitudes have been converted to the HSC filter system.

\section{APPENDIX C: CATALOGUE RELEASE COLUMNS}

Table $\mathrm{C} 1$ provides descriptions of selected columns from our catalogue which will appear alongside other data products released as part of the DES Y3 cosmology analysis at https://des.ncsa.illinois.ed $\mathrm{u} / \mathrm{releases.}$ 
Table C1. Selected Deep Field Catalogue Columns. Note: names ending ' $*$ ' show that the quantity is available for UGRIZJHKs filter bands; names ending ' $* *$ ' show that the quantity is available for redshift values from $z=0.01$ to $z=8$. Full details will be available upon release at https://des.ncsa.illinois.edu/releases.

\begin{tabular}{|c|c|c|}
\hline Catalogue Column & Units & Description \\
\hline ID & - & Object identifier \\
\hline TILENAME & - & DECam field and chip number string \\
\hline RA & Deg & Equatorial coordinates $(\mathrm{J} 2000)$ \\
\hline DEC & Deg & Equatorial coordinates $(\mathrm{J} 2000)$ \\
\hline BDF_FLUX_* & $3.63 \mathrm{nJy}$ & Bulge + disc model flux with $\mathrm{AB}$ zero-point $=30$ \\
\hline BDF_FLUX_ERR_* & $3.63 \mathrm{nJy}$ & Bulge + disc model flux uncertainty \\
\hline BDF_FLUX_DERED_CALIB_* & $3.63 \mathrm{nJy}$ & Corrected bulge + disc model flux with $\mathrm{AB}$ zero-point $=30$ \\
\hline BDF_FLUX_ERR_DERED_CALIB_* & $3.63 \mathrm{nJy}$ & Corrected bulge + disc model flux uncertainty \\
\hline BDF_MAG_DERED_CALIB_* & AB mag & Corrected bulge + disc model magnitude \\
\hline BDF_MAG_ERR_DERED_CALIB_* & $\mathrm{AB}$ mag & Corrected bulge + disc model magnitude uncertainty \\
\hline BDF_T & $\operatorname{arcsec}$ & Pre-seeing bulge + disc model size \\
\hline BDF_T_RATIO & - & Bulge + disc model axial ratio \\
\hline BDF_FRACDEV & - & Fraction of light contained in the bulge model component \\
\hline PSF_FLUX_* & $3.63 \mathrm{nJy}$ & PSF model flux with $\mathrm{AB}$ zero-point $=30$ \\
\hline PSF_FLUX_ERR_* & $3.63 \mathrm{nJy}$ & PSF model flux uncertainty \\
\hline PSF_MAG_* & $\mathrm{AB}$ mag & PSF model magnitude \\
\hline PSF_MAG_ERR_* & AB mag & PSF model magnitude uncertainty \\
\hline PSF_MAG_DERED_CALIB_* & AB mag & Corrected PSF model magnitude \\
\hline PSF_MAG_ERR_DERED_CALIB_* & $\mathrm{AB}$ mag & Corrected PSF model magnitude uncertainty \\
\hline PSF_T & $\operatorname{arcsec}$ & Model PSF size \\
\hline GAP_FLUX_* & $3.63 \mathrm{nJy}$ & Gaussian aperture flux with $\mathrm{AB}$ zero-point $=30$ \\
\hline GAP_FLUX_ERR_* & $3.63 \mathrm{nJy}$ & Gaussian aperture flux uncertainty \\
\hline EBV_SFD98 & - & $E(B-V)$ value from Schlegel et al. (1998) \\
\hline MASK_FLAGS & - & Flag containing mask information for DECam data: Unmasked sources $=0$ \\
\hline MASK_FLAGS_NIR & - & Flag containing mask information for VIRCam data: Unmasked sources $=0$ \\
\hline FLAGS & - & Flag identifier for DECam data \\
\hline FLAGSTR & - & Flag information string for DECam data \\
\hline FLAGS_NIR & - & Flag identifier for VIRCam data \\
\hline FLAGSTR_NIR & - & Flag information string for VIRCam data \\
\hline FOF_ID & - & Friends-of-friends group identifier \\
\hline FOF_SIZE & - & Number of objects in friends-of-friends group \\
\hline Z_P & - & Maximum posterior redshift value \\
\hline Z_PEAK & - & Mean redshift of the most probable redshift peak \\
\hline $\mathrm{Z} \_\mathrm{MC}$ & - & Random draw from probability distribution function \\
\hline PDZ_** & - & Redshift probability density at $z=* *$ \\
\hline KNN_CLASS & - & $\begin{array}{l}\text { Star-galaxy classification: Galaxy }=1, \text { Star }=2, \text { Ambiguous }=3 \text {, } \\
\text { Unclassified }=0\end{array}$ \\
\hline
\end{tabular}


${ }^{1}$ Department of Astronomy, University of Geneva, ch. d'Ecogia 16, CH-1290 Versoix, Switzerland

${ }^{2}$ Centerfor Cosmology and Astro-Particle Physics, The Ohio State University, Columbus, $\mathrm{OH}$ 43210, USA

${ }^{3}$ Kavli Institute for Particle Astrophysics \& Cosmology, PO Box 2450, Stanford University, Stanford, CA 94305, USA

${ }^{4}$ Department of Astronomy, University of Illinois at Urbana-Champaign, 1002 W. Green Street, Urbana, IL 61801, USA

${ }^{5}$ National Center for Supercomputing Applications, 1205 West Clark St, Urbana, IL 61801, USA

${ }^{6}$ Brookhaven National Laboratory, Bldg 510, Upton, NY 11973, USA

${ }^{7}$ Department of Physics, University of Oxford, Denys Wilkinson Building, Keble Road, Oxford OX1 3RH, UK

${ }^{8}$ Jodrell Bank Center for Astrophysics, School of Physics and Astronomy, University of Manchester, Oxford Road, Manchester, M13 9PL, UK

${ }^{9}$ Department of Physics and Astronomy, University of Pennsylvania, Philadelphia, PA 19104, USA

${ }^{10}$ Centro de Investigaciones Energéticas, Medioambientales y Tecnológicas (CIEMAT), Madrid, Spain

${ }^{11}$ Fermi National Accelerator Laboratory, PO Box 500, Batavia, IL 60510, USA

${ }^{12}$ Argonne National Laboratory, 9700 South Cass Avenue, Lemont, IL 60439, USA

${ }^{13}$ Institute of Astronomy, University of Cambridge, Madingley Road, Cambridge CB3 OHA, UK

${ }^{14}$ Kavli Institute for Cosmology, University of Cambridge, Madingley Road, Cambridge CB3 OHA, UK

${ }^{15}$ Physics Department, University of Wisconsin-Madison, 2320 Chamberlin Hall, 1150 University Avenue Madison, WI 53706-1390, USA

${ }^{16}$ SLAC National Accelerator Laboratory, Menlo Park, CA 94025, USA

${ }^{17}$ George P. and Cynthia Woods Mitchell Institute for Fundamental Physics and Astronomy, and Department of Physics and Astronomy, Texas A\&M University, College Station, TX 77843, USA

${ }^{18}$ University of Nottingham, School of Physics and Astronomy, Nottingham NG7 2RD, UK

${ }^{19}$ School of Mathematics and Physics, University of Queensland, Brisbane, QLD 4072, Australia

${ }^{20}$ Department of Physics, Carnegie Mellon University, Pittsburgh, Pennsylvania 15312, USA

${ }^{21}$ Department of Astronomy and Astrophysics, University of Chicago, Chicago, IL 60637, USA

${ }^{22}$ Kavli Institute for Cosmological Physics, University of Chicago, Chicago, IL 60637, USA

${ }^{23}$ Santa Cruz Institute for Particle Physics, Santa Cruz, CA 95064, USA

${ }^{24}$ Jet Propulsion Laboratory, California Institute of Technology, 4800 Oak Grove Dr., Pasadena, CA 91109, USA

${ }^{25}$ Department of Physics, Stanford University, 382 Via Pueblo Mall, Stanford, CA 94305, USA

${ }^{26}$ Department of Physics, The Ohio State University, Columbus, OH 43210, USA

${ }^{27}$ Department of Applied Mathematics and Theoretical Physics, University of Cambridge, Cambridge CB3 OWA, UK

${ }^{28}$ Department of Physics, University of Michigan, Ann Arbor, MI 48109, USA

${ }^{29}$ Department of Astrophysical Sciences, Princeton University, Peyton Hall, Princeton, NJ 08544, USA

${ }^{30}$ Department of Physics, ETH Zurich, Wolfgang-Pauli-Strasse 16, CH-8093 Zurich, Switzerland

${ }^{31}$ Department of Physics, Duke University Durham, NC 27708, USA

${ }^{32}$ Cerro Tololo Inter-American Observatory, NSF's National Optical-Infrared Astronomy Research Laboratory, Casilla 603, La Serena, Chile

${ }^{33}$ Departamento de Física Matemática, Instituto de Física, Universidade de São Paulo, CP 66318, São Paulo, SP 05314-970, Brazil
${ }^{34}$ Laboratório Interinstitucional de e-Astronomia - LIneA, Rua Gal. José Cristino 77, Rio de Janeiro, RJ 20921-400, Brazil

${ }^{35}$ Institute of Cosmology and Gravitation, University of Portsmouth, Portsmouth PO1 3FX, UK

${ }^{36}$ CNRS, UMR 7095, Institut d'Astrophysique de Paris, F-75014 Paris, France

${ }^{37}$ Sorbonne Universités, UPMC Univ Paris 06, UMR 7095, Institut d'Astrophysique de Paris, F-75014 Paris, France

${ }^{38}$ Department of Physics and Astronomy, Pevensey Building, University of Sussex, Brighton BN1 9QH, UK

${ }^{39}$ Department of Physics \& Astronomy, University College London, Gower Street, London WC1E 6BT, UK

${ }^{40}$ Instituto de Astrofisica de Canarias, E-38205 La Laguna, Tenerife, Spain

${ }^{41}$ Universidad de La Laguna, Dpto. Astrofisica, E-38206 La Laguna, Tenerife, Spain

${ }^{42}$ Institut de Física d'Altes Energies (IFAE), The Barcelona Institute of Science and Technology, Campus UAB, 08193 Bellaterra, Barcelona, Spain

${ }^{43}$ Institut d'Estudis Espacials de Catalunya (IEEC), E-08034 Barcelona, Spain

${ }^{44}$ Institute of Space Sciences (ICE, CSIC), Campus UAB, Carrer de Can

Magrans, s/n, E-08193 Barcelona, Spain

${ }^{45}$ INAF - Osservatorio Astronomico di Trieste, via G. B. Tiepolo 11, I-34143 Trieste, Italy

${ }^{46}$ Institute for Fundamental Physics of the Universe, Via Beirut 2, I-34014 Trieste, Italy

${ }^{47}$ Observatório Nacional, Rua Gal. José Cristino 77, Rio de Janeiro, RJ 20921-400, Brazil

${ }^{48}$ Department of Astronomy, University of California, Berkeley, 501 Campbell Hall, Berkeley, CA 94720, USA

${ }^{49}$ Department of Physics, IIT Hyderabad, Kandi, Telangana 502285, India

${ }^{50}$ Faculty of Physics, Ludwig-Maximilians-Universität, Scheinerstr 1, D81679 Munich, Germany

${ }^{51}$ Department of Astronomy/Steward Observatory, University of Arizona, 933 North Cherry Avenue, Tucson, AZ 85721-0065, USA

${ }^{52}$ Institute of Theoretical Astrophysics, University of Oslo. PO Box 1029 Blindern, NO-0315 Oslo, Norway

${ }^{53}$ Instituto de Fisica Teorica UAM/CSIC, Universidad Autonoma de Madrid, E-28049 Madrid, Spain

${ }^{54}$ Department of Astronomy, University of Michigan, Ann Arbor, MI 48109 , USA

${ }^{55}$ Center for Astrophysics | Harvard \& Smithsonian, 60 Garden Street, Cambridge, MA 02138, USA

${ }^{56}$ Australian Astronomical Optics, Macquarie University, North Ryde, NSW 2113, Australia

${ }^{57}$ Lowell Observatory, 1400 Mars Hill Rd, Flagstaff, AZ 86001, USA

${ }^{58}$ Department of Astronomy, The Ohio State University, Columbus, $\mathrm{OH}$ 43210, USA

${ }^{59}$ Radcliffe Institute for Advanced Study, Harvard University, Cambridge, MA 02138, USA

${ }^{60}$ Institució Catalana de Recerca i Estudis Avançats, E-08010 Barcelona, Spain

${ }^{61}$ Max Planck Institute for Extraterrestrial Physics, Giessenbachstrasse, D85748 Garching, Germany

${ }^{62}$ School of Physics and Astronomy, University of Southampton, Southampton SO17 1BJ, UK

${ }^{63}$ Computer Science and Mathematics Division, Oak Ridge National Laboratory, Oak Ridge, TN 37831, USA

${ }^{64}$ Universitäts-Sternwarte, Fakultät für Physik, Ludwig-Maximilians Universität München, Scheinerstr 1, D-81679 München, Germany

${ }^{65}$ Institute for Astronomy, University of Edinburgh, Edinburgh EH9 3HJ, UK

This paper has been typeset from a $\mathrm{T}_{\mathrm{E}} \mathrm{X} / \mathrm{L} \mathrm{T} \mathrm{E} \mathrm{X}$ file prepared by the author. 